\title{
Scaling diagram for the localization length at a band edge
}

\author{
Christian Sadel, Hermann Schulz-Baldes \\ Mathematisches Institut, Universität Erlangen-Nürnberg, Germany
}

\begin{abstract}
A weak-coupling scaling diagram for the Lyapunov exponent and the integrated density of states near a band edge of a random Jacobi matrix is obtained. The analysis is based on the use of a Fokker-Planck operator describing the drift-diffusion of the Prüfer phases.
\end{abstract}

\section{Main result and short overview}

This work considers one-dimensional discrete random Schrödinger operators, typically given by a periodic background operator and a weakly-coupled random potential. The periodic (nonrandom) operator has a band structure. In the vicinity of a band edge, we present a rigorous pertubation theory in the coupling constant and the energy for the Lyapunov exponent and the density of states. This leads to a new and rich scaling diagram describing these two selfaveraging quantities at a band edge.

The scaling behavior at energies within a band has been well understood for a long time. For sake of concreteness, let us focus on the Anderson model $H=\Delta+\lambda V$ given as the sum of the discrete Laplacian $\Delta$ and a disordered potential $V=\sum_{n \in \mathbb{Z}} v_{n}|n\rangle\langle n|$ coupled with a (small) coupling constant $\lambda \geq 0$. Thouless [Tho found a perturbative formula for the Lyapunov exponent (inverse localization length) at an energy $E=2 \cos (k)$ in the band spectrum of $\Delta$ :

$$
\gamma_{\lambda}(E)=\frac{\mathbf{E}_{\sigma}\left(v_{\sigma}^{2}\right)}{8 \sin ^{2}(k)} \lambda^{2}+\mathcal{O}\left(\lambda^{3}\right)
$$

where $v_{\sigma}$ is one of the identically distributed and centered entries of $V$ and $\mathbf{E}_{\sigma}$ denotes the expectation value. A rigorous proof was provided by Pastur and Figotin using the Prüfer phases, also called the Dyson-Schmidt variables [PF]. This amounts to justifying the so-called random phase approximation, stating that the Prüfer phases are distributed according to the Lebesgue measure on the unit circle. A further generalization was given in [JSS]. One feature of (1) are the singularities at the band edges $k=0, \pi$ of the unperturbed operator $\Delta$. In fact, the control on the error terms in (11) breaks down at those energies, as does the random phase 
approximation. The latter also happens at the band center, namely for $k=\frac{\pi}{2}, \frac{3 \pi}{2}$; this leads to anomalies in the perturbative formula (11) first found by Kappus and Wegner [KW], and consecutively analyzed by several authors [DG, BK, CK, SVW]. A perturbative formula as (1) for the band center with a control on the error terms was proven only recently [S1].

This paper considers the band edges and shows how the $\lambda^{2}$ is modified, leading to the new scaling diagram near a band edge. Parts of this diagram were already given by Derrida and Gardner [DG]. These authors actually found the correct scaling in the parabolic regime of Theorem 1 below, but the wrong prefactor ( $c f$. the comment at the end of Section 8). Moreover, they could not give a better justification of their scaling Ansatz than that it leads to a differential equation they could solve. Our more conceptual approach shows why the scaling is natural in the situation considered in [DG]. It exhibits a far richer scaling behavior near a band edge and also allows to rigorously control the higher order corrections. Moreover, if one wants to give a perturbative proof of uniform positivity of the Lyapunov exponent in an energy interval around a band edge, all of the scaling behaviors considered below are needed.

Let us now describe the main result in more detail. We consider a one-parameter family of random Jacobi matrices $\left(H_{\lambda, \omega}\right)_{\lambda \geq 0}$ given as the sum of an $L$-periodic background operator $H_{0}=H_{0, \omega}$ and a random perturbation $H_{\lambda, \omega}-H_{0}$ which is linear in the (small) coupling constant $\lambda$. More precisely, for every fixed configuration $\omega$, the operator $H_{\lambda, \omega}$ acts on $\psi \in \ell^{2}(\mathbb{Z})$ as

$$
H_{\lambda, \omega}|n\rangle=t_{\lambda, \omega}(n+1)|n+1\rangle+v_{\lambda, \omega}(n)|n\rangle+t_{\lambda, \omega}(n)|n-1\rangle, \quad n \in \mathbb{Z},
$$

where the coefficients $t_{\lambda, \omega}(n)>0$ and $v_{\lambda, \omega}(n) \in \mathbb{R}$ are constructed as described in the following: let $\left(\hat{t}_{1}, \ldots, \hat{t}_{L}, \hat{v}_{1}, \ldots, \hat{v}_{L}\right)$ be given real constants with $\hat{t}_{l}>0$ for $l=1, \ldots, L$; set $\Sigma=[-1,1]^{2 L}$ so that each $\sigma \in \Sigma$ is of the form $\sigma=\left(\tilde{t}_{1}(\sigma), \ldots, \tilde{t}_{L}(\sigma), \tilde{v}_{1}(\sigma), \ldots, \tilde{v}_{L}(\sigma)\right)$; then $\Omega=\Sigma^{\mathbb{Z}} \times\{1, \ldots, L\}$ is the configuration space and to each $\omega=\left(\left(\sigma_{m}\right)_{m \in \mathbb{Z}}, k\right) \in \Omega$ there are associated sequences

$$
t_{\lambda, \omega}(k-1+L m+l)=\hat{t}_{l}+\lambda \tilde{t}_{l}\left(\sigma_{m}\right), \quad v_{\lambda, \omega}(k-1+L m+l)=\hat{v}_{l}+\lambda \tilde{v}_{l}\left(\sigma_{m}\right),
$$

which for $\lambda$ sufficiently small satisfy $t_{\lambda, \omega}(n)>0$; these sequences define $H_{\lambda, \omega}$ by (2). In order to make $\left(H_{\lambda, \omega}\right)_{\lambda \geq 0}$ into a family of random operators, we equip $\Omega$ with a probability measure $\mathbf{P}=\mathbf{p}^{\mathbb{Z}} \times \frac{1}{L} \sum_{l=1}^{L} \delta_{l}$ where $\mathbf{p}$ is a probability measure on $\Sigma$. Expectation values w.r.t. $\mathbf{P}$ and $\mathbf{p}$ will be denoted by $\mathbf{E}$ and $\mathbf{E}_{\sigma}$. We suppose that $\mathbf{E}_{\sigma}\left(\tilde{t}_{l}(\sigma)\right)=\mathbf{E}_{\sigma}\left(\tilde{v}_{l}(\sigma)\right)=0$.

For every fixed energy $E \in \mathbb{R}$ and coupling parameter $\lambda$, there are two self-averaging quantities of interest, namely the integrated density of states (IDS) $\mathcal{N}_{\lambda}(E)$ and the Lyapunov exponent (or inverse localization length) $\gamma_{\lambda}(E)$. The definitions will be recalled in Section 4 below. The periodic operator $H_{0}=H_{0, \omega}$ has a band structure and we are interested in the scaling of the IDS and Lyapunov exponent at one of its band edges $E_{b}$ (band touching excluded). In order to state the precise result, we need to introduce two quantities. Let $\mathcal{T}_{\lambda, \sigma}^{E}$ be the random transfer matrix at energy $E$ over a unit cell of length $L$ (see Section 2 for the explicit formula). Then set

$$
x=\left.\partial_{E} \operatorname{Tr}\left(\mathcal{T}_{0, \sigma}^{E}\right)\right|_{E=E_{b}}, \quad x_{\sigma}=\left.\partial_{\lambda} \operatorname{Tr}\left(\mathcal{T}_{\lambda, \sigma}^{E_{b}}\right)\right|_{\lambda=0} .
$$


Theorem 1 Let $E_{b}$ be a band edge (band touching excluded) of the periodic background operator $H_{0}=H_{0, \omega}$ of a family of random Jacobi matrices $\left(H_{\lambda, \omega}\right)_{\lambda \geq 0}$. The perturbation is supposed to be non-trivial in the sense that $x_{\sigma}$ does not vanish $\mathbf{p}$-almost surely. Then the scaling near the band edge is

$$
\mathcal{N}_{\lambda}\left(E_{b}+\epsilon \lambda^{\eta}\right)=\mathcal{N}_{0}\left(E_{b}\right)+A \lambda^{\alpha}+\mathcal{O}\left(\lambda^{\alpha+\delta}\right), \quad \gamma_{\lambda}\left(E_{b}+\epsilon \lambda^{\eta}\right)=B \lambda^{\beta}+\mathcal{O}\left(\lambda^{\beta+\delta}\right),
$$

where $A, B, \alpha, \beta$ and $\delta>0$ depend on $\epsilon \in \mathbb{R}$ and $\eta>0$ as described in the following and resumed in Figure 1.

(i) (Elliptic regime) Let $\eta<\frac{4}{3}$ and let the sign of $\epsilon \neq 0$ be such that $E_{b}+\epsilon \lambda^{\eta}$ is inside the band of $H_{0}$. For the case of the Lyapunov exponent, we also suppose $\eta>\frac{4}{5}$. Then

$$
\alpha=\frac{\eta}{2}, \quad \beta=2-\eta
$$

and

$$
A=\operatorname{sgn}(\epsilon) \frac{\sqrt{|\epsilon x|}}{L \pi}, \quad B=\frac{1}{8 L} \frac{\mathbf{E}_{\sigma}\left(\left|x_{\sigma}\right|^{2}\right)}{|\epsilon x|} .
$$

(ii) (Parabolic regime) Let $\eta=\frac{4}{3}$, then

$$
\alpha=\beta=\frac{2}{3}
$$

and $A=A(\epsilon)$ and $B=B(\epsilon)$ are given by integrals written out explicitly in Section 8 . For $\eta>\frac{4}{3}, A$ and $B$ are independent of $\epsilon$, namely the result is the same as for $\epsilon=0$.

(iii) (Hyperbolic regime) Let $\frac{4}{5}<\eta<\frac{4}{3}$ and let the sign of $\epsilon \neq 0$ be such that $E_{b}+\epsilon \lambda^{\eta}$ is outside the band of $H_{0}$. Then

$$
\alpha>\frac{\eta}{2}, \quad \beta=\frac{\eta}{2}
$$

and

$$
B=\sqrt{|\epsilon x|} .
$$

The result is summarized in Figure 1. All the error estimates, in particular, the value of $\delta$, are controlled more explicitely and given in Section 8. As will become more clear below, the terms elliptic, parabolic and hyperbolic regimes reflect the nature of the corresponding averaged transfer matrix to lowest order in $\lambda$. Let us point out that we do not provide the asymptotics for the IDS in the hyperbolic regime, but only give an upper bound on it. Actually the IDS between the Lifshitz tails and the band edge of the periodic operator is exponentially small in $\lambda$, a fact that results from a more delicate large deviation behavior. This will be dealt with elsewhere. The Lifshitz tails themselves were analyzed in [S2].

The proof of Theorem 1 begins (in Section 2) with an adequate basis change on the transfer matrices. A band edge is characterized by the fact that the modulus of the trace of the transfer matrix is 2 . If there is no band touching, the transfer matrix is then not diagonalizable and its normal form is hence a Jordan block (Appendix A). The action of a Jordan block on projective 


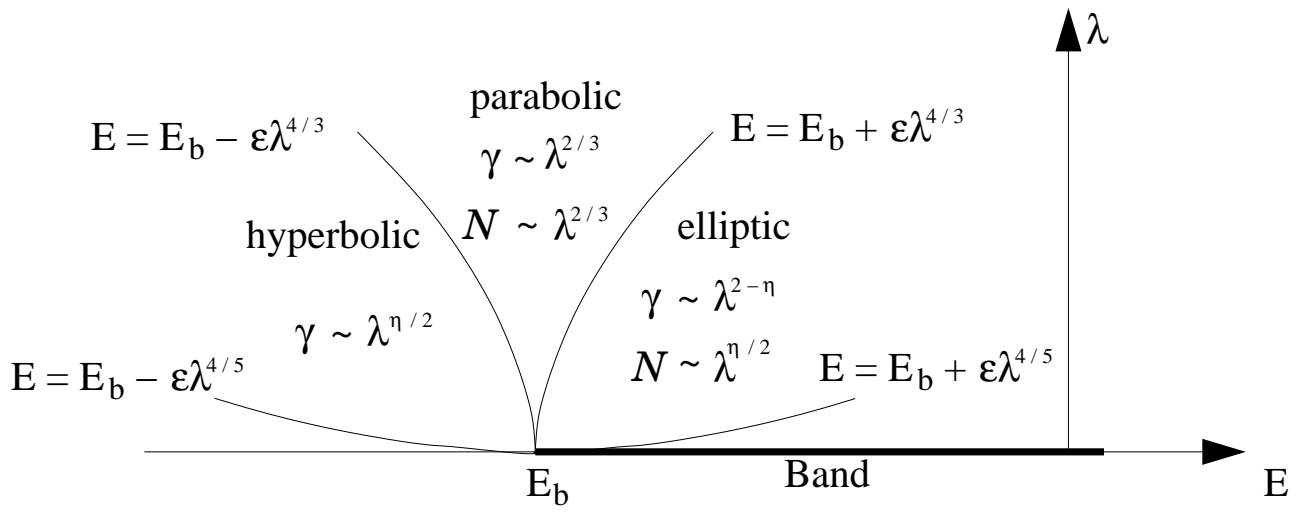

Figure 1: Phase diagram

space (in fact, identified with the Prüfer phases) has exactly one fixed point, which is unstable under random pertubations induced by the randomness in the Hamiltonian. In order to analyse the associated invariant distribution on projective space, a further basis change blowing up the vicinity of the fixed point is necessary. If this is done adequately, a formal perturbative calculation typically leads to a differential equation for the invariant distribution.

Instead of studying the concrete form of the random matrices obtained in Section 2 after the various basis changes, we rather choose to single out the more general concept of an anomaly of a family of random matrices ( $c f$. Section 3 ). The term anomaly is chosen by reference to the center of band anomaly in the one-dimensional Anderson model as studied by Kappus and Wegner [KW]. The latter is a special case which is analyzed in a prior work [S1]. The formalism to calculate the Lyapunov exponents and rotation numbers at a general anomaly is built up in Section 4. In particular, it turns out that one needs to evaluate certain Birkhoff sums for the perturbative calculation of these quantities. The perturbative evaluation of these Birkhoff sums directly leads to a differential operator on projective space (Propositions 1 and 22). In some situations this operator is of first order, and consequently the anomaly is called first order as well (this is dealt with in Section 5). However, in the more interesting cases treated in Sections 6 and 7, the differential operator is of second order and of the Fokker-Planck type. The latter operator was already used in [S1] in order to study the center of band anomaly, but here one is confronted with the supplementary difficulty that its ellipticity is destroyed at a band edge. Thus a thorough analysis of the corresponding singularities is needed. Section 6 deals with the Fokker-Planck operator and its groundstate, while Section 7 shows how it is used to calculate the Birkhoff sums. Both sections heavily depend on Appendix B, where inhomogeneous singular first order ordinary differential equations are studied in detail. Sections 3 up to 7 are kept slightly more general than needed for the proof of Theorem 1 as completed in Section 8 , but we hope that this stresses the structural aspects of the analysis. There may possibly also be further applications.

Acknowledgment: We are thankful to Andreas Knauf for numerous comments and discussions. This work was support by the DFG. 


\section{Normal form of transfer matrix at a band edge}

In this section we motivate and carry out the basis changes on the transfer matrices in the vicinity of a band edge. The final normal forms obtained in the various regimes will then motivate the definition of the term anomaly in the next section.

First, one rewrites the Schrödinger equation $H_{\lambda, \omega} \psi=E \psi$, with $E \in \mathbb{R}, \psi \in \ell^{2}(\mathbb{Z})$ and $H_{\lambda, \omega}$ as given in (2), in the standard way using transfer matrices (e.g. [JSS]). Due to the periodicity of $H_{0}$ it is convenient to introduce the transfer matrix over $L$ sites associated to one configuration $\sigma \in \Sigma$ of the disorder on these sites:

$$
\mathcal{T}_{\lambda, \sigma}^{E}=\prod_{l=1}^{L} \mathcal{T}^{E}\left(\hat{t}_{l}+\lambda \tilde{t}_{l}(\sigma), \hat{v}_{l}+\lambda \tilde{v}_{l}(\sigma)\right), \quad \mathcal{T}^{E}(t, v)=\left(\begin{array}{cc}
(E-v) t^{-1} & -t \\
t^{-1} & 0
\end{array}\right) .
$$

Note that $\mathcal{T}^{E}(t, v) \in \mathrm{SL}(2, \mathbb{R})$ and hence $\mathcal{T}_{\lambda, \sigma}^{E} \in \mathrm{SL}(2, \mathbb{R})$. If $E_{b}$ is a band edge of $H_{0}$, then $\operatorname{Tr}\left(\mathcal{T}_{0, \sigma}^{E_{b}}\right)= \pm 2$ and, because there is no band touching, $\partial_{E} \operatorname{Tr}\left(\mathcal{T}_{0, \sigma}^{E_{b}}\right) \neq 0$. One can show that necessarily $\mathcal{T}_{0, \sigma}^{E_{b}}$ has only one eigenvector (see Appendix A). Hence one can find a basis change $N \in \mathrm{SL}(2, \mathbb{R})$ such that $N \mathcal{T}_{0, \sigma}^{E_{b}} N^{-1}$ is a Jordan block with eigenvalue either 1 or -1 . This elementary fact was already efficiently used for the analysis of the Lifshitz tails [S2]. Using this basis change, one can write the full energy rescaled transfer matrix as follows:

$$
N \mathcal{T}_{\lambda, \sigma}^{E_{b}+\epsilon \lambda^{\eta}} N^{-1}= \pm \exp \left(\left(\begin{array}{cc}
0 & \pm 1 \\
0 & 0
\end{array}\right)+\sum_{k \geq 1} \lambda^{\eta_{k}} Q_{\eta_{k}, \sigma}\right)
$$

Here the exponents $\eta_{k}$ are in $\mathbb{N}+\eta \mathbb{N}$, are put into increasing order $\eta_{k}<\eta_{k+1}$ and the $Q_{\eta_{k}, \sigma}$ are in the Lie algebra $\mathrm{sl}(2, \mathbb{R})$. Hence the lowest terms are $\lambda^{\eta} Q_{\eta, \sigma}$ or $\lambda Q_{1, \sigma}$, pending on the value of $\eta$. Let us note that $Q_{k \eta, \sigma}$ are independent of $\sigma$ unless $k \eta \in \mathbb{N}$. Also, $\mathbf{E}_{\sigma}\left(Q_{1, \sigma}\right)=0$ unless there is a $k$ such that $k \eta=1$. Each combination of the signs can occur, the one inside the exponential indicates if it is a lower $(+$ sign) or an upper $(-$ sign $)$ bandedge. For sake of concreteness, we choose both signs positive.

A few further remarks on the $Q_{\eta_{k}, \sigma}$ will be relevant later on. Because there is no band touching at $E_{b}$, for $\epsilon \neq 0$ one gets

$$
0 \neq\left.\epsilon \partial_{E} \operatorname{Tr}\left(N \mathcal{T}_{0, \sigma}^{E} N^{-1}\right)\right|_{E=E_{b}}=\operatorname{Tr}\left(Q_{\eta, \sigma}\left(\begin{array}{ll}
1 & 1 \\
0 & 1
\end{array}\right)\right)=\epsilon x,
$$

where the first identity follows from the definition (5), Duhamel's formula and the cyclicity of the trace, and the second one defines $x$. By the same calculation (with $\epsilon=0$ )

$$
\left.\partial_{\lambda} \operatorname{Tr}\left(N \mathcal{T}_{\lambda, \sigma}^{E_{b}} N^{-1}\right)\right|_{\lambda=0}=\operatorname{Tr}\left(Q_{1, \sigma}\left(\begin{array}{ll}
1 & 1 \\
0 & 1
\end{array}\right)\right)=x_{\sigma}
$$

the latter by definition of the centered random variable $x_{\sigma}$. Of course this expression is centered because the perturbation is centered. However, the assumption in Theorem 1 on the noninvariance of the band edge under perturbation translates into

$$
\mathbf{E}\left(\left[\operatorname{Tr}\left(Q_{1, \sigma}\left(\begin{array}{ll}
1 & 1 \\
0 & 1
\end{array}\right)\right)\right]^{2}\right)>0 .
$$


It turns out (see Section 4 or [JSS]) that one needs to study the random dynamical system given by random iteration of the natural action of the transfer matrices $N \mathcal{T}_{\lambda, \sigma}^{E_{b}+\epsilon \lambda^{\eta}} N^{-1}$ on $S_{\pi}^{1} \cong \mathbb{R} / \pi \mathbb{Z}$, given by (see Section 4 for details)

$$
e_{\theta_{n}}= \pm \frac{N \mathcal{T}_{\lambda, \sigma_{n}}^{E_{b}+\epsilon \lambda^{\eta}} N^{-1} e_{\theta_{n-1}}}{\left\|N \mathcal{T}_{\lambda, \sigma_{n}}^{E_{b}+\epsilon \lambda^{\eta}} N^{-1} e_{\theta_{n-1}}\right\|}, \quad e_{\theta}=\left(\begin{array}{c}
\cos (\theta) \\
\sin (\theta)
\end{array}\right)
$$

At $\lambda=0$, the action is given by the graph of Figure 2, It has an unstable parabolic fixed

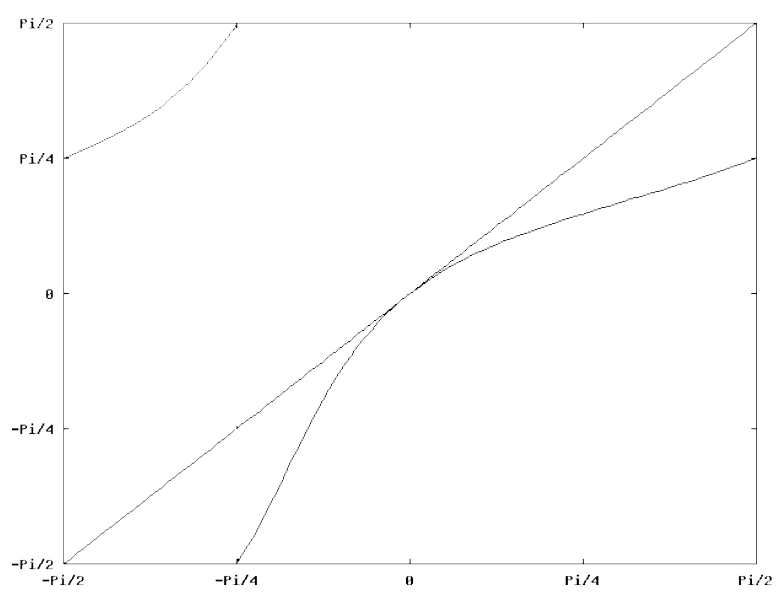

Figure 2: Dynamics at $\lambda=0$ at lower band edge, i.e. + sign in (5).

point at $\theta=0$, which is being approached from the left under the dynamics without passing it though. For $\lambda>0$, the dynamics is the same to lowest order $\lambda^{0}$, but the random perturbations of higher order may allow the dynamics to cross through $\theta=0$. Nevertheless, the angles $\theta_{n}$ are close to $\theta=0$ for most $n$ and thus their distribution is more and more concentrated in the vicinity of $\theta=0$ as $\lambda$ tends to 0 and, in fact, converges weakly to a Dirac peak. In order to extract the shape of the non-trivial distribution it is necessary to rescale the neighborhood of $\theta=0$ with an adequate power of $\lambda$. This is done by conjugation with

$$
N_{\lambda, \delta}=\left(\begin{array}{cc}
\lambda^{\delta} & 0 \\
0 & 1
\end{array}\right) \text {. }
$$

The conjugation has the following effect on $2 \times 2$ matrices:

$$
N_{\lambda, \delta}\left(\begin{array}{ll}
a & b \\
c & d
\end{array}\right) N_{\lambda, \delta}^{-1}=\lambda^{-\delta}\left(\begin{array}{ll}
0 & 0 \\
c & 0
\end{array}\right)+\left(\begin{array}{ll}
a & 0 \\
0 & d
\end{array}\right)+\lambda^{\delta}\left(\begin{array}{ll}
0 & b \\
0 & 0
\end{array}\right) .
$$

One deduces from (5)

$$
N_{\lambda, \delta} N \mathcal{T}_{\lambda, \sigma}^{E_{b}+\epsilon \lambda^{\eta}} N^{-1} N_{\lambda, \delta}^{-1}=\exp \left(N_{\lambda, \delta}\left(\begin{array}{ll}
0 & 1 \\
0 & 0
\end{array}\right) N_{\lambda, \delta}^{-1}+\sum_{k \geq 1} \lambda^{\eta_{k}} N_{\lambda, \delta} Q_{\eta_{k}, \sigma} N_{\lambda, \delta}^{-1}\right) .
$$


Note that the powers of $\lambda$ appearing in the exponential now lie in $\mathbb{N}+\eta \mathbb{N}+\{-\delta, 0, \delta\}$, but none is negative for $\delta<\min \{1, \eta\}$. The following three contributions of low order in $\lambda$ turn out to be relevant:

$X_{\delta, 1}=\lambda^{\delta}\left(\begin{array}{ll}0 & 1 \\ 0 & 0\end{array}\right), X_{\delta, 2}=\lambda^{\eta-\delta} \lim _{\lambda^{\prime} \rightarrow 0} \lambda^{\prime} N_{\lambda^{\prime}, 1} Q_{\eta, \sigma} N_{\lambda^{\prime}, 1}^{-1}, \quad X_{\delta, \sigma, 3}=\lambda^{1-\delta} \lim _{\lambda^{\prime} \rightarrow 0} \lambda^{\prime} N_{\lambda^{\prime}, 1} Q_{1, \sigma} N_{\lambda^{\prime}, 1}^{-1}$,

where the notation reflects that $Q_{\eta, \sigma}$ is independent of $\sigma$. By equations (9) and (6), $X_{\delta, 2}$ has a non-vanishing constant entry only in the lower left corner unless $\epsilon=0$. Similarly by (9) and (7), $X_{\delta, \sigma, 3}$ has a centered random entry in the lower left corner with positive variance, while all the other entries vanish. It is helpful to think of $X_{\delta, 1}$ and $X_{\delta, 2}$ as drift terms in the action (8), and of $X_{\delta, \sigma, 3}$ as generator of a diffusion. Now we blow up the parabolic fixed point by augmenting $\delta$ until the drift $X_{\delta, 1}$ is of same order of magnitude as either the drift $X_{\delta, 2}$ (then $\delta=\frac{\eta}{2}$ ) or the diffusive force (variance) of $X_{\delta, \sigma, 3}$ (then $2(1-\delta)=\delta$ so that $\delta=\frac{2}{3}$ ). Hence we choose

$$
\delta=\min \left\{\frac{\eta}{2}, \frac{2}{3}\right\} .
$$

Explicitely, for $\eta<\frac{4}{3}$, one has with this choice of $\delta$

$$
N_{\lambda, \delta} N \mathcal{T}_{\lambda, \sigma}^{E_{b}+\epsilon \lambda^{\eta}} N^{-1} N_{\lambda, \delta}^{-1}=\exp \left(\lambda^{\frac{\eta}{2}}\left(\begin{array}{cc}
0 & 1 \\
\epsilon x & 0
\end{array}\right)+\lambda^{1-\frac{\eta}{2}}\left(\begin{array}{cc}
0 & 0 \\
x_{\sigma} & 0
\end{array}\right)+\lambda^{\eta} Q_{\eta}^{\prime}+\lambda Q_{1, \sigma}^{\prime}+\mathcal{O}\right),
$$

where $\mathcal{O}=\mathcal{O}\left(\lambda^{\frac{3 \eta}{2}}, \lambda^{1+\frac{\eta}{2}}\right)$ and the specific form of $Q_{\eta}^{\prime}$ and $Q_{1, \sigma}^{\prime}$ will not be of importance for the asymptotics of Theorem 1. For $\eta>1$, the second term is of lower order than the first, but as it is centered, we shall see that it does not enter into the asymptotics of the IDS and the Lyapunov exponent because its variance is smaller than $\mathcal{O}\left(\lambda^{\frac{\eta}{2}}\right)$. Hence (10) is an anomaly of first order in the sense of the following section. In the case $\eta=\frac{4}{3}$,

$$
N_{\lambda, \delta} N \mathcal{T}_{\lambda, \sigma}^{E_{b}+\epsilon \lambda^{\eta}} N^{-1} N_{\lambda, \delta}^{-1}=\exp \left(\lambda^{\frac{1}{3}}\left(\begin{array}{cc}
0 & 0 \\
x_{\sigma} & 0
\end{array}\right)+\lambda^{\frac{2}{3}}\left(\begin{array}{cc}
0 & 1 \\
\epsilon x & 0
\end{array}\right)+\mathcal{O}(\lambda)\right) .
$$

This is actually the same formula as (10), but it was written out again in order to clearly exhibit the so-called anomaly of second order (see Definition 2 below), namely the variance of the first centered term is precisely of the same order of magnitude as the expectation value of the second. If $\eta>\frac{4}{3}$, one obtains the same formula with $\epsilon=0$, but with an additional error $\operatorname{term} \mathcal{O}\left(\lambda^{\eta-\frac{2}{3}}\right)$.

\section{Definition of anomalies}

Let us consider families $\left(T_{\lambda, \sigma}\right)_{\lambda \in \mathbb{R}, \sigma \in \Sigma}$ of matrices in $\mathrm{SL}(2, \mathbb{R})$ depending on a random variable $\sigma$ in some probability space $(\Sigma, \mathbf{p})$ as well as a real coupling parameter $\lambda$. In order to avoid technicalities, we suppose that $T_{\lambda, \sigma}$ has compact support for small $\lambda$. Furthermore we assume that the dependence on $\lambda$ can be expanded in some power series with non-negative exponents (not necessarily integers). Later on, we shall choose $T_{\lambda, \sigma}$ to be the matrices in (10) and (11). 
Definition 1 The value $\lambda=0$ is an anomaly of the family $\left(T_{\lambda, \sigma}\right)_{\lambda \in \mathbb{R}, \sigma \in \Sigma}$ if for all $\sigma \in \Sigma$ and a sign that may depend on $\sigma \in \Sigma$ :

$$
T_{0, \sigma}= \pm 1
$$

In order to further classify the anomalies, let us suppose that for a fixed $\lambda$ - and $\sigma$-independent basis change $M \in \mathrm{SL}(2, \mathbb{R})$ to be chosen later, the transfer matrix is of the following form:

$$
M T_{\lambda, \sigma} M^{-1}= \pm \exp \left(\sum_{k \geq 1} \lambda^{\eta_{k}} P_{\eta_{k}, \sigma}\right) .
$$

Here $P_{\eta_{k}, \sigma} \in \operatorname{sl}(2, \mathbb{R}), \eta_{k}>0$ for $k \in \mathbb{N}$ and $K$, such that $\eta_{j}<\eta_{k}$ if $j<k, \eta_{K}=2 \eta_{1}$, $\eta_{K+1} \leq \eta_{1}+\eta_{2}$ and $\mathbf{p}\left(P_{\eta_{k}, \sigma}=0\right)<1$ for $k=1, \ldots, K-1$ (which means none of these matrices shall be identically 0 for $\mathbf{p}$-almost all $\sigma$ ).

Definition 2 Let $\left(T_{\lambda, \sigma}\right)_{\lambda \in \mathbb{R}, \sigma \in \Sigma}$ have an anomaly and suppose given the expansion (13) for a fixed basis change $M$. The anomaly is said to be of first order and $\mathcal{K}$ th kind if $\mathcal{K}<K$, $\mathbf{E}\left(P_{\eta_{k}, \sigma}\right)=0$ for $k=1, \ldots, \mathcal{K}-1$ and $\mathbf{E}\left(P_{\eta_{\mathcal{K}}, \sigma}\right)$ is non-vanishing. An anomaly of first order and $\mathcal{K}$ th kind is called elliptic if $\operatorname{det}\left(\mathbf{E}\left(P_{\eta_{\mathcal{K}}, \sigma}\right)\right)>0$, hyperbolic if $\operatorname{det}\left(\mathbf{E}\left(P_{\eta_{\mathcal{K}}, \sigma}\right)\right)<0$ and parabolic if $\operatorname{det}\left(\mathbf{E}\left(P_{\eta_{\mathcal{K}}, \sigma}\right)\right)=0$. Note that all these notions are independent of the choice of $M$.

If $\mathbf{E}\left(P_{\eta_{k}, \sigma}\right)=0$, for $k=1, \ldots, K-1$ (then the variance of $P_{\eta_{1}, \sigma}$ is non-vanishing), then an anomaly is said to be of second order.

It may happen that the transfer matrices have to be modified as follows in order to obtain an anomaly. If there is a mapping $\lambda \mapsto M_{\lambda} \in \mathrm{GL}(2, \mathbb{R})$ for $\lambda>0$ where $M_{\lambda}$ is independent of $\sigma$, such that

$$
\lim _{\lambda \rightarrow 0} M_{\lambda} T_{\lambda, \sigma} M_{\lambda}^{-1}= \pm \mathbf{1}
$$

then we say that $T_{\lambda, \sigma}$ is transformed to an anomaly by $M_{\lambda}$. Note that the limits $\lim _{\lambda \downarrow 0} M_{\lambda}$ and $\lim _{\lambda \downarrow 0} M_{\lambda}^{-1}$ need not exist.

As argued in the last section, the transfer matrices at a band edge can be transformed into anomalies (10) and (11) of respectively first and second order. The term anomaly first appeared in the work of Kappus and Wegner [KW] on the band center of a one-dimensional Anderson model. Indeed, as discussed in [S1], the square of the transfer matrix at a band edge is an anomaly in the above sense (of second order, if the random potential is centered). More generally, higher powers of transfer matrices are transformed into anomalies for rational quasimomenta, and thus lead to anomalies in higher order perturbation theory [DG]. A more systematic classification of anomalies as in Definition 2 was done in [S1]. However, we felt

it adequate to change the term degree of an anomaly used in [S1] to the present order of an anomaly because it leads to a differential equation of corresponding order.

\section{Phase shift dynamics}

The bijective action $\mathcal{S}_{T}$ of a matrix $T \in \mathrm{SL}(2, \mathbb{R})$ on $S_{\pi}^{1}=\mathbb{R} / \pi \mathbb{Z}=[0, \pi)$ is given by 


$$
e_{\mathcal{S}_{T}(\theta)}= \pm \frac{T e_{\theta}}{\left\|T e_{\theta}\right\|}, \quad e_{\theta}=\left(\begin{array}{c}
\cos (\theta) \\
\sin (\theta)
\end{array}\right), \quad \theta \in S_{\pi}^{1},
$$

with an adequate choice of the sign. This defines a group action, namely $\mathcal{S}_{T T^{\prime}}=\mathcal{S}_{T} \mathcal{S}_{T^{\prime}}$. In particular the map $\mathcal{S}_{T}$ is invertible and $\mathcal{S}_{T}^{-1}=\mathcal{S}_{T^{-1}}$. Note that this is an action on projective space. In order to shorten notations, we write

$$
\mathcal{S}_{\lambda, \sigma}=\mathcal{S}_{M T_{\lambda, \sigma} M^{-1}}
$$

At an anomaly one has $\mathcal{S}_{\lambda, \sigma}(\theta)=\theta+\mathcal{O}(\lambda)$.

Given an initial angle $\theta_{0}$ and iterating this dynamics defines a Markov process $\theta_{n}\left(\omega, \theta_{0}\right)$ on $\Omega=\Sigma^{\mathbb{Z}} \times\{1, \ldots, L\}$, i.e. for $\omega=\left(\left(\sigma_{n}\right)_{n \in \mathbb{Z}}, k\right)$ one defines iteratively

$$
\theta_{0}\left(\omega, \theta_{0}\right)=\theta_{0}, \quad \theta_{n+1}\left(\omega, \theta_{0}\right)=S_{\lambda, \sigma_{n+1}}\left(\theta_{n}\left(\omega, \theta_{0}\right)\right)
$$

In order to shorten notations, we denote $\theta_{n}\left(\omega, \theta_{0}\right)$ by $\theta_{n}$. Under adequate identifications, this corresponds to the dynamics (8). The $\theta_{n}$ are also called modified Prüfer variables.

In order to analyze the dynamics in more detail, some further notations are needed. For $k \in \mathbb{N}$, we define the trigonometric polynomials:

$$
p_{k, \sigma}(\theta)=\Im m\left(\frac{\left\langle v\left|P_{\eta_{k}, \sigma}\right| e_{\theta}\right\rangle}{\left\langle v \mid e_{\theta}\right\rangle}\right), \quad v=\frac{1}{\sqrt{2}}\left(\begin{array}{c}
1 \\
-\imath
\end{array}\right) .
$$

More explicitly, in terms of the matrix elements one obtains

$$
P_{\eta_{k}, \sigma}=\left(\begin{array}{cc}
a_{\eta_{k}, \sigma} & b_{\eta_{k}, \sigma} \\
c_{\eta_{k}, \sigma} & -a_{\eta_{k}, \sigma}
\end{array}\right) \Longrightarrow p_{k, \sigma}(\theta)=c_{\eta_{k}, \sigma} \cos ^{2}(\theta)-b_{\eta_{k}, \sigma} \sin ^{2}(\theta)-a_{\eta_{k}, \sigma} \sin (2 \theta) .
$$

Now starting from the identity

$$
e^{2 \imath \mathcal{S}_{\lambda, \sigma}(\theta)}=\frac{\left\langle v\left|M T_{\lambda, \sigma} M^{-1}\right| e_{\theta}\right\rangle}{\left\langle\bar{v}\left|M T_{\lambda, \sigma} M^{-1}\right| e_{\theta}\right\rangle}
$$

the definition (13) and the identity $\left\langle v \mid e_{\theta}\right\rangle=\frac{1}{\sqrt{2}} e^{\imath \theta}$, one can verify that

$\mathcal{S}_{\lambda, \sigma}(\theta)=\theta+\Im m\left(\sum_{k=1}^{K} \lambda^{\eta_{k}} \frac{\left\langle v\left|P_{\eta_{k}, \sigma}\right| e_{\theta}\right\rangle}{\left\langle v \mid e_{\theta}\right\rangle}+\lambda^{2 \eta_{1}}\left[\frac{\left\langle v\left|P_{\eta_{1}, \sigma}^{2}\right| e_{\theta}\right\rangle}{2\left\langle v \mid e_{\theta}\right\rangle}-\frac{\left\langle v\left|P_{\eta_{1}, \sigma}\right| e_{\theta}\right\rangle^{2}}{2\left\langle v \mid e_{\theta}\right\rangle^{2}}\right]\right)+\mathcal{O}\left(\lambda^{\eta_{K+1}}\right)$.

As one readily verifies that

$$
P_{\eta_{1}, \sigma}^{2}=-\operatorname{det}\left(P_{\eta_{1}, \sigma}\right) \mathbf{1}, \quad \Im m\left(\frac{\left\langle v\left|P_{\eta_{1}, \sigma}\right| e_{\theta}\right\rangle^{2}}{\left\langle v \mid e_{\theta}\right\rangle^{2}}\right)=-p_{1, \sigma} \partial_{\theta} p_{1, \sigma}(\theta),
$$

it follows that

$$
\mathcal{S}_{\lambda, \sigma}(\theta)=\theta+\sum_{k=1}^{K} \lambda^{\eta_{k}} p_{k, \sigma}(\theta)+\frac{1}{2} \lambda^{2 \eta_{1}} p_{1, \sigma} \partial_{\theta} p_{1, \sigma}(\theta)+\mathcal{O}\left(\lambda^{\eta_{K+1}}\right) .
$$


As one has $\exp \left(\sum \lambda^{\eta_{k}} P_{\eta_{k}, \sigma}\right)^{-1}=\exp \left(-\sum \lambda^{\eta_{k}} P_{\eta_{k}, \sigma}\right)$ the same procedure leads to

$$
\mathcal{S}_{\lambda, \sigma}^{-1}(\theta)=\theta-\sum_{k=1}^{K} \lambda^{\eta_{k}} p_{k, \sigma}(\theta)+\frac{1}{2} \lambda^{2 \eta_{1}} p_{\eta_{1}, \sigma} \partial_{\theta} p_{\eta_{1}, \sigma}(\theta)+\mathcal{O}\left(\lambda^{\eta_{K+1}}\right) .
$$

Our main interest is the perturbative calculation of the Lyapunov exponent $\gamma(\lambda)$ and rotation number $\mathcal{R}(\lambda)$ associated to the random family of matrices $\left(T_{\lambda, \sigma_{n}}\right)_{n \geq 1}$ which are defined by

$$
\gamma(\lambda)=\lim _{N \rightarrow \infty} \frac{1}{N} \mathbf{E} \sum_{n=0}^{N-1} \mathbf{E}_{\sigma} \log \left(\left\|M T_{\lambda, \sigma} M^{-1} e_{\theta_{n}}\right\|\right)
$$

and

$$
\mathcal{R}(\lambda)=\frac{1}{\pi} \lim _{N \rightarrow \infty} \frac{1}{N} \mathbf{E} \sum_{n=1}^{N} \mathbf{E}_{\sigma} \varphi_{\lambda, \sigma}\left(\theta_{n}\right) .
$$

where $\varphi_{\lambda, \sigma}: S_{\pi}^{1} \rightarrow \mathbb{R}$ is the phase shift given by $\varphi_{\lambda, \sigma}(\theta)=\mathcal{S}_{\lambda, \sigma}(\theta)-\theta$. If $T_{\lambda, \sigma}=\tilde{N} \mathcal{T}_{\lambda, \sigma}^{E+\epsilon \lambda^{\eta}} \tilde{N}^{-1}$ where the latter is the transfer matrix as given in (4) associated to a random Jacobi matrix $H_{\lambda, \omega}$ with an $L$-periodic background operator defined in (2) and $\tilde{N}$ is an arbitrary basis change such as the ones used in (10) and (11), then the Lyapunov exponent $\gamma_{\lambda}(E)$ (inverse localization length) and $\operatorname{IDS} \mathcal{N}_{\lambda}(E)$ of the random Jacobi matrix are

$$
\gamma_{\lambda}\left(E+\epsilon \lambda^{\eta}\right)=\frac{1}{L} \gamma(\lambda), \quad \mathcal{N}_{\lambda}\left(E+\epsilon \lambda^{\eta}\right)=-\frac{1}{L} \mathcal{R}(\lambda) \bmod \frac{1}{L} .
$$

The first identity follows immediately from the definition; the second is a consequence of the oscillation theorem and the gap labelling for the periodic operator (see e.g. [JSS] for details). In both of the expressions (20) and (21), one can now expand each summand w.r.t. $\lambda$.

Lemma 1 Set

$$
\alpha_{\eta, \sigma}=\left\langle v\left|P_{\eta, \sigma}\right| v\right\rangle, \quad \beta_{\eta, \sigma}=\left\langle\bar{v}\left|P_{\eta, \sigma}\right| v\right\rangle, \quad \gamma_{\eta, \sigma}=\left\langle\left.\bar{v}|| P_{\eta, \sigma}\right|^{2} \mid v\right\rangle
$$

as well as

$$
\delta_{\eta_{1}, \eta_{2}, \sigma}=\frac{1}{2}\left\langle\bar{v}\left|\left(P_{\eta_{1}, \sigma}+P_{\eta_{1}, \sigma}^{*}\right) P_{\eta_{2}, \sigma}+\left(P_{\eta_{2}, \sigma}+P_{\eta_{2}, \sigma}^{*}\right) P_{\eta_{1}, \sigma}\right| v\right\rangle .
$$

Then $p_{\eta, \sigma}(\theta)=\Im m\left(\alpha_{\eta, \sigma}-\beta_{\eta, \sigma} e^{2 \imath \theta}\right)$. Furthermore, with errors of order $\mathcal{O}\left(\lambda^{3 \eta_{1}}\right)$,

$$
\begin{aligned}
\log \left(\left\|M T_{\lambda, \sigma} M^{-1} e_{\theta}\right\|\right) & =\Re e \sum_{k \geq 1}\left(\lambda^{\eta_{k}} \beta_{\eta_{k}, \sigma} e^{2 \imath \theta}+\frac{\lambda^{2 \eta_{k}}}{2}\left(\left|\beta_{\eta_{k}, \sigma}\right|^{2}+\gamma_{\eta_{k}, \sigma} e^{2 \imath \theta}-\beta_{\eta_{k}, \sigma}^{2} e^{4 \imath \theta}\right)\right)+ \\
& +\Re e \sum_{k_{1}<k_{2}}\left(\lambda^{\eta_{k_{1}}+\eta_{k_{2}}}\left(\beta_{\eta_{k_{1}}, \sigma} \overline{\beta_{\eta_{k_{2}}, \sigma}}+\delta_{\eta_{k_{1}}, \eta_{k_{2}}, \sigma} e^{2 \imath \theta}-\beta_{\eta_{k_{1}}, \sigma} \beta_{\eta_{k_{2}}, \sigma} e^{4 \imath \theta}\right)\right),
\end{aligned}
$$

and up to errors of order $\mathcal{O}\left(\lambda^{\eta_{1}+\eta_{2}}\right)$

$$
\varphi_{\lambda, \sigma}(\theta)=\Im m \sum_{k \geq 1}\left(\lambda^{\eta_{k}}\left(\alpha_{\eta_{k}, \sigma}-\beta_{\eta_{k}, \sigma} e^{2 \imath \theta}\right)+\frac{1}{2} \lambda^{2 \eta_{k}}\left(-2 \alpha_{\eta_{k}, \sigma} \beta_{\eta_{k}, \sigma} e^{2 \imath \theta}+\beta_{\eta_{k}, \sigma}^{2} e^{4 \imath \theta}\right)\right) \text {. }
$$


Proof. This follows from straightforward algebra using

$$
\left\langle e_{\theta}|T| e_{\theta}\right\rangle=\frac{1}{2} \operatorname{Tr}(T)+\Re e\left(\langle\bar{v}|T| v\rangle e^{2 \imath \theta}\right), \quad \operatorname{Tr}\left(\left|P_{\eta, \sigma}\right|^{2}+P_{\eta, \sigma}^{2}\right)=4\left|\beta_{\eta, \sigma}\right|^{2},
$$

as well as the identities $\operatorname{Tr}\left(P_{\eta, \sigma}\right)=0$ and $\left\langle\bar{v}\left|P_{\eta, \sigma}^{2}\right| v\right\rangle=0$.

Once these formulas are replaced in (20) and (21), one hence needs to consider Birkhoff sums of the type

$$
I_{N}(f)=\frac{1}{N} \mathbf{E} \sum_{n=0}^{N-1} f\left(\theta_{n}\right), \quad I(f)=\lim _{N \rightarrow \infty} I_{N}(f)
$$

for $\pi$-periodic functions $f$. For $\gamma(\lambda)$ and $\mathcal{R}(\lambda)$ one actually only needs the functions $e^{22 \theta}$ and $e^{4 \imath \theta}$. These Birkhoff sums have to be evaluated perturbatively in $\lambda$ with a rigorous control on the error terms. For this purpose, one needs to know the distribution of the $\theta_{n}$ as generated by the random dynamics (18). This is the main focus of the next sections.

\section{$5 \quad$ First order anomalies}

It turns out that it is easiest to calculate the Birkhoff sums $I_{N}(f)$ in case of an elliptic first order anomaly of $\mathcal{K}$ th kind. As then $\operatorname{det}\left(\mathbf{E}\left(P_{\eta_{\mathcal{K}}, \sigma}\right)\right)>0$, one can choose the basis change $M$ in (13) such that

$$
\mathbf{E}\left(P_{\eta_{\mathcal{K}}, \sigma}\right)=\left(\begin{array}{cc}
0 & -\mu \\
\mu & 0
\end{array}\right)
$$

with $\mu \neq 0$. Hence $\mathbf{E}\left(p_{\mathcal{K}, \sigma}(\theta)\right)=\mu$.

Proposition 1 Let $T_{\lambda, \sigma}$ have an elliptic anomaly of first order and $\mathcal{K}$ th kind and suppose that $\mathbf{E}\left(p_{\mathcal{K}, \sigma}(\theta)\right)=\mu$. Then for any $f \in C^{1}\left(S_{\pi}^{1}\right)$, one has

$$
I_{N}(f)=\int_{0}^{\pi} \frac{\mathrm{d} \theta}{\pi} f(\theta)+\mathcal{O}\left(\lambda^{\eta_{\mathcal{K}^{\prime}}-\eta_{\mathcal{K}}},\left(\lambda^{\eta \mathcal{K}} N\right)^{-1}\right)
$$

with $\mathcal{K}^{\prime}=\min \{\tilde{\mathcal{K}}, K\}>\mathcal{K}$ where $\tilde{\mathcal{K}}$ is such that $P_{\eta_{\tilde{\mathcal{K}}}}$ is the first uncentered term in (13) after $P_{\eta_{\mathcal{K}}}$.

Proof. Because $I_{N}(f)=c+I_{N}(f-c)$ for $c=\int_{0}^{\pi} \mathrm{d} \theta f(\theta) / \pi$, we may assume that $\int_{0}^{\pi} \mathrm{d} \theta f(\theta)=0$. Then $f$ has an antiderivative $F \in C^{2}\left(S_{\pi}^{1}\right)$. Using the Taylor expansion and $2 \eta_{1}=\eta_{K}$,

$F\left(\mathcal{S}_{\lambda, \sigma}(\theta)\right)=F\left(\theta+\sum_{k=1}^{K} \lambda^{\eta_{k}} p_{k, \sigma}(\theta)+\mathcal{O}\left(\lambda^{\eta_{K+1}}\right)\right)=F(\theta)+F^{\prime}(\theta) \sum_{k=1}^{K-1} \lambda^{\eta_{k}} p_{k, \sigma}(\theta)+\mathcal{O}\left(\lambda^{\eta_{K}}\right)$.

From this one deduces after taking expectation and summing over $n$ :

$$
I_{N}(F)=I_{N}(F)+\mu \lambda^{\eta_{\mathcal{K}}} I_{N}(f)+\mathcal{O}\left(\lambda^{\eta_{\mathcal{K}^{\prime}}}, N^{-1}\right)
$$


As $\mu \neq 0$ this proves the estimate.

Concerning hyperbolic and parabolic first order anomalies, we do not provide an exhaustive treatment, but rather present some procedures on how these anomalies can (possibly) be transformed into a more accessible anomaly such as one of second order. This applies, in particular, to the hyperbolic first order anomaly (10) with $\epsilon x$ negative, corresponding to the hyperbolic regime in Theorem 1, For a hyperbolic first order anomaly of $\mathcal{K}$ th kind, let us first choose $M$ such that

$$
\mathbf{E}\left(P_{\eta_{\mathcal{K}}, \sigma}\right)=\left(\begin{array}{cc}
-\mu & 0 \\
0 & \mu
\end{array}\right), \quad \mu>0 .
$$

Then to lowest order in $\lambda, \theta=0$ is the unstable and $\theta=\frac{\pi}{2}$ the stable fixed point of (the averaged dynamics of) $\mathcal{S}_{\lambda, \sigma}$. Like in (11), we use the $\lambda$-dependent basis change $N_{\lambda, \delta}$. Let $\chi$ be the smallest exponent such that $\mathbf{E}\left(c_{\chi, \sigma}^{2}\right)>0$. First suppose that $\mathbf{E}\left(c_{\xi, \sigma}\right)=0$ for all $\xi<\frac{1}{2} \eta_{\mathcal{K}}+\chi$, then choose $\delta=\chi-\frac{1}{2} \eta_{\mathcal{K}}>0$ so that $2(\chi-\delta)=\eta_{\mathcal{K}}$. One obtains

$$
N_{\lambda, \delta} M T_{\lambda, \sigma} M^{-1} N_{\lambda, \delta}^{-1}= \pm \exp \left[\lambda^{\frac{1}{2} \eta_{\mathcal{K}}}\left(\begin{array}{cc}
0 & 0 \\
c_{\chi, \sigma} & 0
\end{array}\right)+\ldots+\lambda^{\eta_{\mathcal{K}}}\left(\begin{array}{cc}
a_{\eta_{\mathcal{K}}, \sigma} & b_{\eta_{\mathcal{K}}-\delta, \sigma} \\
c_{\eta_{\mathcal{K}}+\delta, \sigma} & -a_{\eta_{\mathcal{K}}, \sigma}
\end{array}\right)+\ldots\right]
$$

where, moreover, $\mathbf{E}\left(b_{\eta_{\mathcal{K}}-\delta, \sigma}\right)=0$ and $\mathbf{E}\left(a_{\eta_{\mathcal{K}}, \sigma}\right)=-\mu<0$. This is a second order anomaly for which the Birkhoff sums are analyzed in Theorem 4 below. If, on the other hand, there is a $\xi<\frac{1}{2} \eta_{\mathcal{K}}+\chi$ such that $\mathbf{E}\left(c_{\xi, \sigma}\right) \neq 0$ and $\xi$ is the smallest such exponent, choose $\delta=\xi-\eta_{\mathcal{K}}>0$ so that

$$
N_{\lambda, \delta} M T M^{-1} N_{\lambda, \delta}^{-1}= \pm \exp \left[\lambda^{\zeta}\left(\begin{array}{cc}
a_{\zeta, \sigma} & 0 \\
c_{\zeta+\delta, \sigma} & -a_{\zeta, \sigma}
\end{array}\right)+\ldots+\lambda^{\eta_{\mathcal{K}}}\left(\begin{array}{cc}
a_{\eta_{\mathcal{K}}, \sigma} & b_{\eta_{\mathcal{K}}-\delta, \sigma} \\
c_{\xi, \sigma} & -a_{\eta_{\mathcal{K}}, \sigma}
\end{array}\right)\right]
$$

where $\zeta$ is either $\eta_{1}$ or $\chi-\delta$. For both cases we have $2 \zeta>\eta_{\mathcal{K}}$. As $\mathbf{E}\left(b_{\eta_{\mathcal{K}}-\delta, \sigma}\right)=0$, one thus has again a hyperbolic first degree anomaly. Repeating the procedure, one may now be in the above advantageous case.

A parabolic first order anomaly of $\mathcal{K}$ th kind can be treated just as a the band edge (which is parabolic to 0th order) in Section 2. One first chooses $M$ to be a rotation matrix, such that the parabolic fixed point is $\theta=0$, namely $\mathbf{E}\left(P_{\eta_{\mathcal{K}}, \sigma}\right) e_{0}=0$, and then considers the matrices $N_{\lambda, \delta} M T_{\lambda, \sigma} M^{-1} N_{\lambda, \delta}^{-1}$. Let again $\chi$ be the smallest exponent such that $\mathbf{E}\left(c_{\chi, \sigma}^{2}\right)>0$ and let $\xi$ be the smallest exponent such that $\mathbf{E}\left(c_{\xi, \sigma}\right) \neq 0$. If $\xi \geq \frac{4}{3} \chi+\frac{1}{3} \eta_{\mathcal{K}}$ and $\mathbf{E}\left(a_{\eta, \sigma}\right)=0$ for all $\eta<\frac{2}{3}\left(\chi+\eta_{\mathcal{K}}\right)$, then one chooses $\delta=\frac{1}{3}\left(2 \chi-\eta_{\mathcal{K}}\right)>0$ in order to get a second order anomaly

$$
\pm \exp \left[\lambda^{\frac{1}{3}\left(\chi+\eta_{\mathcal{K}}\right)}\left(\begin{array}{cc}
a_{\chi-\delta, \sigma} & 0 \\
c_{\chi, \sigma} & -a_{\chi-\delta, \sigma}
\end{array}\right)+\ldots+\lambda^{\frac{2}{3}\left(\chi+\eta_{\mathcal{K}}\right)}\left(\begin{array}{cc}
a_{\eta_{\mathcal{K}}+\delta, \sigma} & b_{\eta_{\mathcal{K}, \sigma}} \\
c_{\eta_{\mathcal{K}}+2 \delta, \sigma} & -a_{\eta_{\mathcal{K}}+\delta, \sigma}
\end{array}\right)+\ldots\right]
$$

\section{Fokker-Planck operator of a second order anomaly}

In order to calculate $I_{N}(f)$ at a second order anomaly, we first refine the proof of Proposition 1 . This naturally leads to an associated differential operator, as shown in the next proposition. The remainder of this section analyzes the properties of these operators. 
Proposition 2 Let the random family $\left(T_{\lambda, \sigma}\right)_{\sigma \in \Sigma}$ have a second order anomaly and $F \in C^{3}\left(S_{\pi}^{1}\right)$. Then, for

$$
f=\mathbf{E}\left(p_{1, \sigma}^{2}\right) F^{\prime \prime}+\left(2 \mathbf{E}\left(p_{K, \sigma}\right)+\mathbf{E}\left(p_{1, \sigma} \partial_{\theta} p_{1, \sigma}\right)\right) F^{\prime},
$$

one has

$$
I_{N}(f)=\mathcal{O}\left(\lambda^{\tilde{\eta}-\eta_{K}},\left(\lambda^{\eta_{K}} N\right)^{-1}\right) .
$$

with $\tilde{\eta}=\min \left\{\eta_{\tilde{K}}, \eta_{1}+\eta_{2}\right\}$ where $\tilde{K}$ is the first uncentered term in (13) after $P_{\eta_{K}, \sigma}$.

Proof. A Taylor expansion implies

$$
\begin{aligned}
& F\left(S_{\lambda, \sigma}(\theta)\right)=F\left(\theta+\sum_{k=1}^{\tilde{K}-1} \lambda^{\eta_{k}} p_{k, \sigma}(\theta)+\frac{1}{2} \lambda^{2 \eta_{1}} p_{1, \sigma} \partial_{\theta} p_{1, \sigma}(\theta)+\mathcal{O}\left(\lambda^{\tilde{\eta}}\right)\right) \\
& =F(\theta)+F^{\prime}(\theta) \sum_{k=1}^{\tilde{K}-1} \lambda^{\eta_{k}} p_{k, \sigma}(\theta)+\frac{1}{2} \lambda^{2 \eta_{1}}\left(p_{1, \sigma} \partial_{\theta} p_{1, \sigma}(\theta) F^{\prime}(\theta)+p_{1, \sigma}^{2}(\theta) F^{\prime \prime}(\theta)\right)+\mathcal{O}\left(\lambda^{\tilde{\eta}}\right) .
\end{aligned}
$$

Taking expectation values and summing over $n$, one gets the estimate by the same argument as in Proposition 1 because $\eta_{K}=2 \eta_{1}$.

Let us sketch the further strategy. In view of (29), it is natural to introduce:

$$
p=\mathbf{E}\left(p_{1, \sigma}^{2}\right), \quad q=2 \mathbf{E}\left(p_{K, \sigma}\right)+\mathbf{E}\left(p_{1, \sigma} \partial_{\theta} p_{1, \sigma}\right) .
$$

Both $p$ and $q$ are the trigonometric polynomials of degree 4 on $S_{\pi}^{1}$, namely linear combinations of $e^{ \pm 4 \imath \theta}, e^{ \pm 2 \imath \theta}$ and the constant function. Moreover, $p \geq 0$. Hence $p$ can have at most one zero of order 4 , or two zeros of order 2 . Actually a zero $\hat{\theta}$ of order 4 only appears in the rather special case described next. By applying a rotation matrix $M$ in (13), one may assume $\hat{\theta}=\frac{\pi}{2}$ and then one readily checks that

$$
p(\theta)=\mathbf{E}\left(c_{\eta_{1}, \sigma}^{2}\right) \cos ^{4}(\theta), \quad P_{\eta_{1}, \sigma}=\left(\begin{array}{cc}
0 & 0 \\
c_{\eta_{1}, \sigma} & 0
\end{array}\right) .
$$

This is precisely the situation in (11). Furthermore, let us note that $q$ can have zeros with orders adding up to at most 4 .

Proposition 2 now states that one can control Birkhoff sums of functions which are in the range of the differential operator on $C^{3}\left(S_{\pi}^{1}\right)$ given by

$$
\mathcal{L}=\left(p \partial_{\theta}+q\right) \partial_{\theta} .
$$

The formal adjoint on $L^{2}\left(S_{\pi}^{1}\right)$, also considered as an operator defined on $C^{3}\left(S_{\pi}^{1}\right)$, is given by

$$
\mathcal{L}^{*}=\partial_{\theta}\left(\partial_{\theta} p-q\right) .
$$

The operator $\mathcal{L}^{*}$ is a Fokker-Planck (or forward Kolmogorov) operator on $S_{\pi}^{1}$ describing a driftdiffusion dynamics on $S_{\pi}^{1}$, while $\mathcal{L}$ is the associated backward Kolmogorov operator [Ris]. The 
operator $\mathcal{L}^{*}$ was already used in [S1], but only in the situation of a strictly positive $p$. In the case of the anomaly (11) corresponding to the parabolic regime at a band edge, $p$ has a zero. Hence the ellipticity of $\mathcal{L}^{*}$ is destroyed. This is the main technical problem that has to be dealt with in the present work.

As $\operatorname{Ran}(\mathcal{L}) \subset \operatorname{Ker}\left(\mathcal{L}^{*}\right)^{\perp}$, a necessary condition for controlling the Birkhoff sum $I_{N}(f)$ is hence that $f \in \operatorname{Ker}\left(\mathcal{L}^{*}\right)^{\perp}$. As will shortly be shown in Theorem 2, in many situations $\operatorname{Ker}\left(\mathcal{L}^{*}\right)$ is one-dimensional and spanned by a smooth positive, $L^{1}$-normalized function $\rho$. Let us call $\rho$ the groundstate of the Fokker-Planck operator, even though the spectrum of the FokkerPlanck operator is non-positive. Furthermore, the necessary condition $f \in \operatorname{Ker}\left(\mathcal{L}_{j}^{*}\right)^{\perp}$ combined with $f \in C^{2}\left(S_{\pi}^{1}\right)$ will in most cases turn out (Theorem 3 below) to be sufficient for finding a solution $F \in C^{3}\left(S_{\pi}^{1}\right)$ of the inhomogeneous differential equation (29). Thus one deduces from Proposition 2 that, for $f \in C^{2}\left(S_{\pi}^{1}\right)$,

$$
I_{N}(f)=\int \mathrm{d} \theta \rho(\theta) f(\theta)+\mathcal{O}\left(\lambda^{\eta_{K+1}-\eta_{K}},\left(\lambda^{\eta_{K}} N\right)^{-1}\right) .
$$

In some other situations, relevant for the hyperbolic regime in Theorem 1, the kernel of $\mathcal{L}^{*}$ is given by a Dirac peak $\rho$ (if $\mathcal{L}^{*}$ is considered as an operator on the space of distributions), and the same formula holds.

Before proving the main result on the groundstate $\rho$, let us point out that $\rho$ can be seen as the lowest order formal approximation to the Furstenberg measure $\nu_{\lambda}$ on $S_{\pi}^{1}$ associated to family $\left(T_{\lambda, \sigma}\right)_{\sigma \in \Sigma}$ of random matrices. It is defined by [BL]

$$
\int_{0}^{\pi} \nu_{\lambda}(\mathrm{d} \theta) f(\theta)=\mathbf{E} \int_{0}^{\pi} \nu_{\lambda}(\mathrm{d} \theta) f\left(S_{\lambda, \sigma}(\theta)\right), \quad f \in C\left(S_{\pi}^{1}\right) .
$$

In fact, supposing $\nu_{\lambda}(\mathrm{d} \theta)=\rho_{\lambda}(\theta) \mathrm{d} \theta$, (31) leads to

$$
\mathbf{E}\left(\partial_{\theta} \mathcal{S}_{\lambda, \sigma}^{-1}(\theta) \rho_{\lambda}\left(\mathcal{S}_{\lambda, \sigma}^{-1}(\theta)\right)\right)=\rho_{\lambda}(\theta) .
$$

Furthermore assuming that $\rho_{\lambda}$ is twice differentiable and $\rho_{\lambda}=\rho+\mathcal{O}\left(\lambda^{\eta_{K+1}-\eta_{K}}\right)$, this gives the equation $\mathcal{L}^{*} \rho=0$ (cf. [S1] for further details).

Theorem 2 The Fokker-Planck operator $\mathcal{L}^{*}$ has a unique groundstate $\rho$, which is non-negative, normalized and continuous except possibly in the following cases:

(I) $p$ has a zero $\hat{\theta}$ of order 2 (and possibly another zero) for which, moreover,

$$
\partial_{\theta} p(\hat{\theta})=q(\hat{\theta})=0, \quad \partial_{\theta}^{2} p(\hat{\theta}) \geq \partial_{\theta} q(\hat{\theta}) .
$$

(II) $p$ has a zero $\hat{\theta}$ of order 4 for which, moreover,

$$
\mathbf{E}\left(p_{K, \sigma}(\hat{\theta})\right)=0, \quad \mathbf{E}\left(\partial_{\theta} p_{K, \sigma}(\hat{\theta})\right) \leq 0 .
$$

Furthermore, the groundstate is infinitely differentiable except possibly at one point $\hat{\theta}$, at which $p$ has a zero of order 2 and

$$
\partial_{\theta} p(\hat{\theta})=q(\hat{\theta}), \quad \partial_{\theta}^{2} p(\hat{\theta})<\partial_{\theta} q(\hat{\theta}) .
$$


Let us note that (32) is equivalent to

$$
\mathbf{E}\left(\partial_{\theta} p_{1, \sigma}^{2}(\hat{\theta})\right)=4 \mathbf{E}\left(p_{K, \sigma}(\hat{\theta})\right), \quad \mathbf{E}\left(\partial_{\theta}^{2} p_{1, \sigma}^{2}(\hat{\theta})\right)>4 \mathbf{E}\left(\partial_{\theta} p_{K, \sigma}(\hat{\theta})\right) .
$$

Furthermore, let us point out that whenever there is a point $\hat{\theta}$ which is a zero of both $p$ and $q$, then the Dirac peak $\delta_{\hat{\theta}}$ is a groundstate of $\mathcal{L}^{*}$ if considered as an operator on distributions. This Dirac peak is dynamically stable under the drift-diffusion described by $\mathcal{L}^{*}$ if, moreover,

$$
\partial^{l}\left(\left(\partial_{\theta} p\right)-q\right)(\hat{\theta})>0, \quad l \in\{1,3\} .
$$

This is precisely the case in (I) and (II). A corresponding dual result, namely that the Birkhoff sums $I_{N}(f)$ are equal to $f(\hat{\theta})$ to lowest order, will be proven in Theorem 4 below. Let us also note that there may be two such angles (e.g. if $\left.\mathbf{E}\left(p_{K, \sigma}\right)=0\right)$, in which case the stable groundstate is degenerate. Finally, we encourage the reader to draw the curves of $p$ and $q$ in each of the cases of the proof below, and interprete $p$ as a diffusive force, and $q$ as a drift. This allows to better understand the formal proof, as well as the result itself.

Proof of Theorem 2. The equation $\mathcal{L}^{*} \rho=0$ can be integrated once. Hence one needs to solve

$$
\left(p \partial_{\theta}+\tilde{q}\right) \rho=C, \quad \tilde{q}=\left(\partial_{\theta} p\right)-q,
$$

where the real constant $C$ has to be chosen such that (35) admits a non-negative, $\pi$-periodic, $L^{1}$ normalized solution $\rho$. The equation (35) is locally precisely the type of singular ODE studied in Appendix B, with coefficients which are moreover real analytic. The only supplementary property of (35) is that the inhomogeneity is constant. When writing out solutions, we use similar notations as in the appendix, except that we shall put tildes on $w$ and $W$ in order to distinguish them from the corresponding dual objects appearing in the proof of Theorem 3 below, and that we do not include the constant inhomogeneity $C$ in the definition of $\tilde{W}$ below as done in (47).

If $p>0$ so that there is no singularity and $\mathcal{L}^{*}$ is elliptic, the groundstate $\rho$ can readily be calculated as in [S1]. Set, for some $\tilde{\theta} \in S_{\pi}^{1}$,

$$
\tilde{w}(\theta)=\int_{\tilde{\theta}}^{\theta} \mathrm{d} \xi \frac{\tilde{q}(\xi)}{p(\xi)}, \quad \tilde{W}(\theta)=\int_{\tilde{\theta}}^{\theta} \mathrm{d} \xi \frac{e^{\tilde{w}(\xi)}}{p(\xi)} .
$$

Then

$$
\rho=C_{1} e^{-\tilde{w}}\left(C_{2} \tilde{W}+1\right), \quad C_{2}=\frac{e^{\tilde{w}(\tilde{\theta}+\pi)}-1}{\tilde{W}(\tilde{\theta}+\pi)}, \quad C=C_{1} C_{2},
$$

where one first determines the normalization constant $C_{1}$ which then also fixes $C$ in (35) by the last identity. Note that $\tilde{W}(\theta)>0$ for $\theta>\tilde{\theta}$ so that, in particular, $C_{2}$ is well-defined. Furthermore $C_{2}=0$ if $w$ is $\pi$-periodic. The groundstate $\rho$ is clearly real analytic in this case.

Now let $\hat{\theta}$ be the only zero of $p$. It is either of order 2 or 4 . If $\tilde{q}(\hat{\theta}) \neq 0$, one is locally near $\hat{\theta}$ in the situation of Proposition 3 (iv) or (v). For each $C$ in (35), the smooth integrable solution is unique to one side of $\hat{\theta}$. Continuing it cyclically around $S_{\pi}^{1}$ shows that it is also unique on the other side of $\hat{\theta}$. For $\hat{\theta} \leq \theta<\hat{\theta}+\pi$, this smooth solution is given by

$$
\rho=C e^{-\tilde{w}} \tilde{W},
$$


with $\tilde{w}$ and $\tilde{W}$ defined as in (36) using $\hat{\theta}<\tilde{\theta}<\hat{\theta}+\pi$ in the first equation and $\tilde{\theta}=\hat{\theta}$ or $\tilde{\theta}=\hat{\theta}+\pi$ in the second one, such that the singularities cancel. It only remains to choose $C \neq 0$ (and hence the ODE (35) itself) such that $\rho$ is normalized.

If $p$ has two zeros $\hat{\theta}_{1}$ and $\hat{\theta}_{2}$ of order 2 for which $\tilde{q}\left(\hat{\theta}_{1}\right) \neq 0$ and $\tilde{q}\left(\hat{\theta}_{2}\right) \neq 0$, one has to distinguish two cases. If the signs of $\tilde{q}\left(\hat{\theta}_{1}\right)$ and $\tilde{q}\left(\hat{\theta}_{2}\right)$ are the same, then one proceeds cyclically twice as in the case with one zero of $p$ which was just treated. The solution is as in (38) and $C \neq 0$. If on the other hand $0 \leq \hat{\theta}_{1}<\hat{\theta}_{2}<\pi$ with $\tilde{q}\left(\hat{\theta}_{1}\right)<0$ and $\tilde{q}\left(\hat{\theta}_{2}\right)>0$, then one chooses $C=0$ and sets

$$
\rho(\theta)=\left\{\begin{array}{cc}
C_{3} e^{-\tilde{w}(\theta)} & \hat{\theta}_{1}<\theta<\hat{\theta}_{2}, \\
0 & \text { otherwise },
\end{array}\right.
$$

with $\tilde{w}$ defined as in (36) for some $\tilde{\theta}$ with $\hat{\theta}_{1}<\tilde{\theta}<\hat{\theta}_{2}$ and some normalization constant $C_{3}$. Note that this $\rho$ has no singularities because $\lim _{\theta \downarrow \hat{\theta}_{1}} \tilde{w}(\theta)=+\infty$ and $\lim _{\theta \uparrow \hat{\theta}_{2}} \tilde{w}(\theta)=+\infty$. As shown in Proposition 3 (iv) and (v), $\rho$ is smooth at both $\hat{\theta}_{1}$ and $\hat{\theta}_{2}$.

Now follow the singular cases where $p$ and $q$ have common zeros. The situations (I) and (II) are of this type, but there will appear others for which we then show as stated that there is a unique continuous groundstate. As argued in Appendix B, there can then only be integrable solutions of (35) if the equation is homogeneous, namely $C=0$. One such solution is $\rho=0$, but there may be others. First let $p$ have only one zero $\hat{\theta}$ of order 2 and suppose that $\tilde{q}(\hat{\theta})=0$. Then $\hat{\theta}$ is a zero of order 2 of $\tilde{q}$ if and only if $\partial_{\theta} \tilde{q}(\hat{\theta})=0$, that is $\mathbf{E}\left(\partial_{\theta}^{2} p_{1, \sigma}^{2}(\hat{\theta})\right)=4 \mathbf{E}\left(\partial_{\theta} p_{K, \sigma}(\hat{\theta})\right)$. In this situation, there is a one-parameter family of smooth solutions according to Proposition 3 (i). However, these solutions are typically not periodic and hence this is part of situation (I). If $\hat{\theta}$ is a zero of order 1 of $\tilde{q}$, then Proposition 3 (ii) and (iii) can be used for analyzing the local regularity. Case (iii) applies if $\partial_{\theta} \tilde{q}(\hat{\theta})<0$. Thus one has a two-parameter family of continuous solutions near $\hat{\theta}$; however, one parameter has to assure the periodicity of the solution so that it remains continuous, while the second has to be chosen so that $\rho$ is normalized. Hence the unique continuous groundstate is $\rho=C_{4} e^{-\tilde{w}}$ with an adequate normalization constant $C_{4}$. In the situation (I) when (32) holds with a strict inequality, one has $\partial_{\theta} \tilde{q}(\hat{\theta})>0$ so that Proposition 3(ii) implies that the solution is unique. As $\rho=0$ is one solution, it is the only one.

Next let us consider the case where $p$ has two zeros $\hat{\theta}_{1}$ and $\hat{\theta}_{2}$ of order 2 , and that at least one of them, say $\hat{\theta}_{1}$, is a zero of $\tilde{q}$. If $\partial_{\theta} \tilde{q}\left(\hat{\theta}_{1}\right)>0$ (namely situation (I) again), there is only the zero solution due to Proposition [3(ii).

If $\partial_{\theta} \tilde{q}\left(\hat{\theta}_{1}\right)=0$ and $\tilde{q}\left(\hat{\theta}_{2}\right) \neq 0$, the singularity $\hat{\theta}_{1}$ can due to Proposition 3 (i) only be resolved by choosing $C=0$, but then the zero $\hat{\theta}_{2}$ for which $\tilde{q}\left(\hat{\theta}_{2}\right) \neq 0$ leads to a singularity. Thus there is no continuous solution and this is included in situation (I). If $\partial_{\theta} \tilde{q}\left(\hat{\theta}_{1}\right)<0$ and say $\tilde{q}\left(\hat{\theta}_{2}\right)>0$, then one can appeal to Proposition 3(iii) at $\hat{\theta}_{1}$ and Proposition 3(iv) at $\hat{\theta}_{2}$, and construct the groundstate exactly as in (39). However, $\rho$ might not be differentiable at $\hat{\theta}_{1}$. If $\hat{\theta}_{1}$ and $\hat{\theta}_{2}$ are zeros of both $p$ and $\tilde{q}$, then either $\partial_{\theta} \tilde{q}\left(\hat{\theta}_{1}\right)>0$ or $\partial_{\theta} \tilde{q}\left(\hat{\theta}_{2}\right)>0$. Indeed, $\tilde{q}=\frac{1}{2} \partial_{\theta} p-2 \mathbf{E}\left(p_{K, \sigma}\right)$ and $\mathbf{E}\left(p_{K, \sigma}\right)$ is a trigonometric polynomial of degree 1 and hence can only compensate either $\partial_{\theta} p\left(\hat{\theta}_{1}\right)>0$ or $\partial_{\theta} p\left(\hat{\theta}_{2}\right)>0$. Therefore this corresponds again to situation (I) and there is no normalizable solution. 
In the last remaining case $\hat{\theta}$ is a zero of $p$ of order 4 (hence the only zero of $p$ ) and $\tilde{q}(\hat{\theta})=0$. If $\partial_{\theta} \tilde{q}(\hat{\theta})<0$, which is equivalent to $\mathbf{E}\left(\partial_{\theta} p_{K, \sigma}(\hat{\theta})\right)>0$, Proposition 3(vii) can be applied and the groundstate $\rho=C_{5} e^{-\tilde{w}}$ is smooth. If $\partial_{\theta} \tilde{q}(\hat{\theta})>0$, then Proposition 3(vi) implies that the zero solution is the only solution. If $\partial_{\theta} \tilde{q}(\hat{\theta})=0$ and $\mathbf{E}\left(p_{K, \sigma}\right)=0$ identically, then $\tilde{q}=\frac{1}{2} \partial_{\theta} p$ has a zero at $\hat{\theta}$ of order 3 and $\partial_{\theta}^{3} \tilde{q}(\hat{\theta})>0$, so that Proposition 3 (ii) implies that the zero solution is the only solution. The same holds if $\partial_{\theta} \tilde{q}(\hat{\theta})=0$ and $\mathbf{E}\left(\partial_{\theta}^{2} p_{K, \sigma}(\hat{\theta})\right) \neq 0$ due to Proposition 3 (iv) or (v), applied either to the left or right of $\hat{\theta}$ pending on the sign of $\mathbf{E}\left(\partial_{\theta}^{2} p_{K, \sigma}(\hat{\theta})\right)$. The last three cases are regrouped in situation (II).

\section{Birkhoff sums at second order anomalies}

In this section we calculate the Birkhoff sums $I_{N}(f)$ perturbatively for many second order anomalies. This involves the analysis of the operator $\mathcal{L}$ dual to the Fokker-Planck operator. We will not present a treatment as exhaustive as Theorem 2 for $\mathcal{L}^{*}$ and do not treat the singular cases where $p$ and $q$ have common zeros, except for the case of Theorem 4 below, which is needed for the analysis of a band edge. Comments on the remaining cases are given at the end of the section.

Theorem 3 Let $\left(T_{\lambda, \sigma}\right)_{\sigma \in \Sigma}$ have an anomaly of second order. Suppose $q(\hat{\theta}) \neq 0$ whenever $\mathbf{E}\left(p_{1, \sigma}^{2}(\hat{\theta})\right)=0$. Furthermore let $\rho$ be the groundstate of the Fokker-Planck operator given by Theorem 2. Then, for $f \in C^{2}\left(S_{\pi}^{1}\right)$ one has

$$
I_{N}(f)=\int_{0}^{\pi} \mathrm{d} \theta f(\theta) \rho(\theta)+\mathcal{O}\left(\lambda^{\tilde{\eta}-\eta_{K}},\left(\lambda^{\eta_{K}} N\right)^{-1}\right)
$$

with $\tilde{\eta}=\min \left\{\eta_{\tilde{K}}, \eta_{1}+\eta_{2}\right\}$ where $\tilde{K}$ is the first uncentered term in (13) after $P_{\eta_{K}, \sigma}$. If $\mathbf{E}\left(p_{1, \sigma}^{2}\right)$ has no zero, it is enough to suppose $f \in C^{1}\left(S_{\pi}^{1}\right)$.

Proof. As already argued in Section [6, one can control the Birkhoff sums using Proposition 2 only if $f \in \operatorname{Ker}\left(\mathcal{L}^{*}\right)^{\perp}$. Under the hypothesis stated, the kernel of $\mathcal{L}^{*}$ is one-dimensional by Theorem 2 and spanned by the groundstate $\rho$. Replacing $f$ by $f-\langle\rho \mid f\rangle$, we may now assume that $\int_{0}^{\pi} \mathrm{d} \theta f(\theta) \rho(\theta)=0$ and have to show for such $f$ that the Birkhoff sum $I_{N}(f)$ is of the order stated. This will follow directly from Proposition 2 once we have found $G \in C^{2}\left(S_{\pi}^{1}\right)$ satisfying

$$
\left(p \partial_{\theta}+q\right) G=f, \quad \int_{0}^{\pi} \mathrm{d} \theta G(\theta)=0 .
$$

Indeed, the second identity allows to take an antiderivative $F$ of $G$ which then satisfies (29).

First let us consider the case where $p$ has no zero and $C \neq 0$ in (35). Setting, for some $\tilde{\theta} \in S_{\pi}^{1}$

$$
w(\theta)=\int_{\tilde{\theta}}^{\theta} \mathrm{d} \xi \frac{q(\xi)}{p(\xi)}, \quad W(\theta)=\int_{\tilde{\theta}}^{\theta} \mathrm{d} \xi \frac{e^{w(\xi)}}{p(\xi)} f(\xi),
$$


the solution is given by

$$
G=e^{-w}\left(W+c_{1}\right), \quad c_{1}=\frac{e^{-w(\tilde{\theta}+\pi)} W(\tilde{\theta}+\pi)}{1-e^{-w(\tilde{\theta}+\pi)}},
$$

where $e^{-w(\tilde{\theta}+\pi)} \neq 1$ as this happens if and only if $w$ and $\tilde{w}$ are $\pi$-periodic leading to $C=0$. One readily checks that the first equation in (40) is satisfied and that $G$ is periodic, hence in $C^{2}\left(S_{\pi}^{1}\right)$ because $f \in C^{1}\left(S_{\pi}^{1}\right)$. Furthermore, multiplying the first equation in (40) by $\rho$ and integrating over $S_{\pi}^{1}$ shows that also the second equation holds:

$$
0=\int \rho f=\int \rho\left(p \partial_{\theta}+q\right) G=-\int G\left(\partial_{\theta} p-q\right) \rho=-C \int G(\theta) .
$$

Next, if $p$ has no zeros and $C=0$ in (35), then $C_{2}=0$ in (37) and

$$
\rho=C_{1} e^{-\tilde{w}}=C_{1} \frac{e^{w}}{p}, \quad\left(p \partial_{\theta}+q\right) e^{-w}=0,
$$

so that $W$ defined as in (41) satisfies $W(\tilde{\theta}+\pi)=W(\tilde{\theta})=0$ because $\int \rho f=0$. Hence

$$
G=e^{-w}\left(W+c_{2}\right)
$$

is a (periodic) function in $C^{2}\left(S_{\pi}^{1}\right)$ and, for adequate choice of $c_{2}$, of vanishing integral.

Now let $p$ have one zero $\hat{\theta}$. The hypothesis implies $q(\hat{\theta}) \neq 0$. According to Proposition 3 (iv) or (v), the first equation in (40) has a $C^{2}$-solution in a neighborhood of $\hat{\theta}$, which is unique to one side of $\hat{\theta}$. Continuing this solution around $S_{\pi}^{1}$ one deduces that the solution is unique, and actually given by $G=e^{-w} W$. Due to the argument in the proof of Theorem 2, $C \neq 0$ in (35) in this case, so that by the same argument as above the second equation in (40) is satisfied. The case with two zeros $\hat{\theta}_{1}$ and $\hat{\theta}_{2}$ of $p$ for which $q\left(\hat{\theta}_{1}\right)$ and $q\left(\hat{\theta}_{2}\right)$ have the same sign is treated similarly ( $c f$. the proof of Theorem 2).

Finally we deal with the case of two zeros $0 \leq \hat{\theta}_{1}<\hat{\theta}_{2}<\pi$ of $p$ for which $q\left(\hat{\theta}_{1}\right)>0$ and $q\left(\hat{\theta}_{2}\right)<0$. By (39) , the support of the groundstate is $\left[\hat{\theta}_{1}, \hat{\theta}_{2}\right]$ and hence not all of $S_{\pi}^{1}$. For $\theta \in\left[\hat{\theta}_{1}, \hat{\theta}_{2}\right]$, we choose $\hat{\theta}_{1}<\tilde{\theta}<\hat{\theta}_{2}$ in the first equation of (41) and $\tilde{\theta}=\hat{\theta}_{1}$ in the second equation of (41). Then $G=e^{-w} W$ is a solution in $\left(\hat{\theta}_{1}, \hat{\theta}_{2}\right)$. Due to Propostion 3 (iv) this is the unique solution that can be smoothly extended to the left of $\hat{\theta}_{1}$. Furthermore (42) and $\int \rho f=0$ imply $W\left(\hat{\theta}_{2}\right)=0$, so that this solution can also be smoothly extended through $\hat{\theta}_{2}$ by Propostion 3(v). On both sides one has one free parameter. One is chosen such that $G$ is $\pi$-periodic and the remaining one such that the second equation of (40) is also satisfied. This reflects that $G_{0}=e^{-w} \chi_{\left[\hat{\theta}_{2}, \hat{\theta}_{1}\right]}$ is a smooth positive solution of the homogeneous equation $\left(p \partial_{\theta}+q\right) G_{0}=0$.

The remaining singular cases for which Theorem 2 guarantees existence of a unique groundstate cannot be treated by the techniques of Theorem 3 without further hypothesis on $f$, which seem somewhat unnatural, but reflect the delicate dynamical behavior at such points (e.g. one needs that $f$ and its derivative have a particular behavior near the common zero of $p$ and $q$ ). The next theorem deals with an anomaly corresponding to situation (II) of Theorem 2, albeit with a strict inequality in (33). 
Theorem 4 Let $\left(T_{\lambda, \sigma}\right)_{\sigma \in \Sigma}$ have an anomaly of second order. Suppose that $\hat{\theta}$ is a zero of order 4 of $\mathbf{E}\left(p_{1, \sigma}^{2}\right)$ and a zero of order 1 of $\mathbf{E}\left(p_{K, \sigma}\right)$ and that, moreover, $\mathbf{E}\left(\partial_{\theta} p_{K, \sigma}(\hat{\theta})\right)<0$. Then for $f \in C^{2}\left(S_{\pi}^{1}\right)$

$$
I_{N}(f)=f(\hat{\theta})+\mathcal{O}\left(\lambda^{\tilde{\eta}-\eta_{K}},\left(\lambda^{\eta_{K}} N\right)^{-1}\right)
$$

with $\tilde{\eta}=\min \left\{\eta_{\tilde{K}}, \eta_{1}+\eta_{2}\right\}$ where $\tilde{K}$ is the first uncentered term in (13) after $P_{\eta_{K}, \sigma}$.

Proof. The procedure is exactly as in the proof of Theorem 3. Hence we search for a solution of (40) for a function $f$ satisfying $f(\hat{\theta})=0$. Close to $\hat{\theta}$ the equation $\left(p \partial_{\theta}+q\right) G=f$ has a twoparameter family of solutions due to Proposition 3(vii). One parameter is fixed by requiring $G$ to be periodic. The other reflects that one can, by the same procedure, find a non-vanishing solution $G_{0}$ to $\left(p \partial_{\theta}+q\right) G_{0}=0$. As $G_{0}$ has definite sign, one can use it to normalize $G$ so that also the second equation in (40) is satisfied.

As to extensions of Theorem 4, the case with an equality in the second equation of (33) cannot be treated by the presented technique because $\left(p \partial_{\theta}+q\right) G_{0}=0$ has only the trivial solution by Proposition 3 (iv) or (v). The case with one zero of order 2 corresponding to situation (I) in Theorem 2 can be treated similarly as long as $3 \mathbf{E}\left(\partial_{\theta}^{2} p_{1, \sigma}^{2}(\hat{\theta})\right)<4\left|\mathbf{E}\left(\partial_{\theta} p_{K, \sigma}(\hat{\theta})\right)\right|$, a condition which originates in Proposition 3(iii).

\section{Application to a band edge}

This section contains the proof of Theorem 1, Hence let $T_{\lambda, \sigma}=N_{\lambda, \frac{\eta}{2}} N \mathcal{T}_{\lambda, \sigma}^{E_{b}+\epsilon \lambda^{\eta}} N^{-1} N_{\lambda, \frac{\eta}{2}}^{-1}$ be the anomaly given in (10) or (11). Then (22) allows to calculate the Lyapunov exponent and the IDS, so that one may focus on the calculation of $\gamma(\lambda)$ and $\mathcal{R}(\lambda)$ based on (20), (21) and the expansions given in Lemma 1 .

Let us begin with the elliptic first order regime, hence (10). The adequate basis change assuring (26) is

$$
M=\left(\begin{array}{cc}
1 & 0 \\
0 & -|\epsilon x|^{-\frac{1}{2}}
\end{array}\right)
$$

Then $\left(M N_{\lambda, \delta} N\right) \mathcal{T}_{\lambda, \sigma}^{E_{b}+\epsilon \lambda^{\eta}}\left(M N_{\lambda, \delta} N\right)^{-1}$ is equal to

$$
\exp \left(\lambda^{\frac{\eta}{2}}|\epsilon x|^{\frac{1}{2}}\left(\begin{array}{cc}
0 & -1 \\
1 & 0
\end{array}\right)-\lambda^{1-\frac{\eta}{2}} \frac{x_{\sigma}}{|\epsilon x|^{\frac{1}{2}}}\left(\begin{array}{ll}
0 & 0 \\
1 & 0
\end{array}\right)+\lambda^{\eta} P_{\eta}+\lambda P_{1, \sigma}+\mathcal{O}\left(\lambda^{\frac{3 \eta}{2}}, \lambda^{1+\frac{\eta}{2}}\right)\right)
$$

Here $\mathbf{E}\left(P_{1-\frac{\eta}{2}, \sigma}\right)=0$, and also $\mathbf{E}\left(P_{1, \sigma}\right)=0$. With the notations of Lemma 1, this gives $\beta_{\frac{\eta}{2}}=0$ and $\beta_{1-\frac{\eta}{2}, \sigma}=\imath x_{\sigma}|\epsilon x|^{-\frac{1}{2}} / 2$. Thus Proposition 1 implies $I\left(e^{2 j \imath \theta}\right)=\mathcal{O}\left(\lambda^{\frac{\eta}{2}}, \lambda^{2-\frac{3 \eta}{2}}\right)$ for $j=1,2$. Hence the first 4 terms with powers $\lambda^{\eta_{k}}$ in (23) either vanish or are of higher order, the same holds for the mixed terms $\lambda^{\eta_{k_{1}}+\eta_{k_{2}}}$, the first of the terms with $\lambda^{2 \eta_{k}}$ is also of higher order, but the second one gives the leading order contribution:

$$
\gamma(\lambda)=\lambda^{2-\eta} \frac{\mathbf{E}\left(x_{\sigma}^{2}\right)}{8|\epsilon x|}+\mathcal{O}\left(\lambda^{\frac{3 \eta}{2}}, \lambda^{4-\frac{5 \eta}{2}}\right)
$$


which together with (22) establishes the first claim. Similarly one verifies $\alpha \frac{\eta}{2}=\imath|\epsilon x|^{\frac{1}{2}}$ so that by (24) one finds $\mathcal{R}(\lambda)=\lambda^{\frac{\eta}{2}}|\epsilon x|^{\frac{1}{2}}+\mathcal{O}\left(\lambda^{\eta}, \lambda^{2-\eta}\right)$.

In the hyperbolic regime, the basis change leading to (27) is

$$
M=\left(\begin{array}{cc}
1 & -|\epsilon x|^{-\frac{1}{2}} \\
1 & |\epsilon x|^{-\frac{1}{2}}
\end{array}\right) \text {. }
$$

Then $\left(M N_{\lambda, \frac{\eta}{2}} N\right) \mathcal{T}_{\lambda, \sigma}^{E_{b}+\epsilon \lambda^{\eta}}\left(M N_{\lambda, \frac{\eta}{2}} N\right)^{-1}$ calculated from (10) is equal to

$$
\exp \left(\lambda^{\frac{\eta}{2}}|\epsilon x|^{\frac{1}{2}}\left(\begin{array}{cc}
-1 & 0 \\
0 & 1
\end{array}\right)+\lambda^{1-\frac{\eta}{2}} \frac{x_{\sigma}}{2|\epsilon x|^{\frac{1}{2}}}\left(\begin{array}{cc}
-1 & -1 \\
1 & 1
\end{array}\right)+\lambda^{\eta} P_{\eta}+\lambda P_{1, \sigma}+\mathcal{O}\left(\lambda^{\frac{3 \eta}{2}}, \lambda^{1+\frac{\eta}{2}}\right)\right) .
$$

Now let us apply a further basis change $N_{\lambda, \delta}$ as described in (28), namely in the present situation $\eta_{\mathcal{K}}=\frac{\eta}{2}, \chi=1-\frac{\eta}{2}$ and $\delta=1-\frac{3 \eta}{4}$. Due to (9) the transformed anomaly then becomes of second order:

$$
\exp \left(\lambda^{\frac{\eta}{4}}|4 \epsilon x|^{-\frac{1}{2}}\left(\begin{array}{cc}
0 & 0 \\
x_{\sigma} & 0
\end{array}\right)+\lambda^{\frac{\eta}{2}}|\epsilon x|^{\frac{1}{2}}\left(\begin{array}{cc}
-1 & 0 \\
0 & 1
\end{array}\right)+\lambda^{1-\frac{\eta}{2}} P_{1-\frac{\eta}{2}, \sigma}^{\prime}+\lambda^{2-\frac{5 \eta}{4}} P_{2-\frac{5 \eta}{4}, \sigma}^{\prime}+\mathcal{O}\right),
$$

where $\mathcal{O}=\mathcal{O}\left(\lambda^{\frac{3 \eta}{4}}, \lambda^{\frac{7 \eta}{4}-1}\right)$. Here $P_{1-\frac{\eta}{2}, \sigma}^{\prime}$ and $P_{2-\frac{5 \eta}{4}, \sigma}^{\prime}$ are possibly of lower order than the second term (but not as the first one as $\eta<\frac{4}{3}$ ), but they are both centered so that they do not enter into the asymptotics ( $c f$. Definition 22). The obtained second anomaly can now be dealt with by Theorem 4, implying that $I(f)=f\left(\frac{\pi}{2}\right)+\mathcal{O}\left(\lambda^{\frac{5 \eta}{4}-1}, \lambda^{1-\frac{3 \eta}{4}}\right)$. Furthermore, one calculates $\beta_{\frac{\eta}{4}, \sigma}=-\imath x_{\sigma}|\epsilon x|^{-\frac{1}{2}} / 4, \gamma_{\frac{\eta}{4}, \sigma}=x_{\sigma}^{2}|\epsilon x|^{-1} / 8$ and $\beta_{\frac{\eta}{2}}=-|\epsilon x|^{\frac{1}{2}}$. Replacing into (23) one realizes that the factor of $\lambda^{2 \frac{\eta}{4}}$ vanishes so that

$$
\gamma(\lambda)=\lambda^{\frac{\eta}{2}}|\epsilon x|^{\frac{1}{2}}+\mathcal{O}\left(\lambda^{\frac{7 \eta}{4}-1}, \lambda^{1-\frac{\eta}{4}}\right) .
$$

Concerning the rotation number, one has $\alpha_{\frac{\eta}{2}}=0$ and $\alpha_{\frac{\eta}{4}, \sigma}=\imath x_{\sigma}|\epsilon x|^{-\frac{1}{2}} / 4$. The latter implies that the contribution $\lambda^{2 \frac{\eta}{4}}$ vanishes in (24) due to the $\Im m$. Thus $\mathcal{R}(\lambda)=\mathcal{O}\left(\lambda^{\frac{7 \eta}{4}-1}, \lambda^{1-\frac{\eta}{4}}\right)$. Note that the condition $\frac{7 \eta}{4}-1>\frac{\eta}{2}$ is equivalent to $\eta>\frac{4}{5}$.

Finally let us deal with the parabolic case for which (11) is directly a second order anomaly. The polynomials (30) are explicitly given by

$$
p(\theta)=\mathbf{E}\left(x_{\sigma}^{2}\right) \cos ^{4}(\theta), \quad q(\theta)=\epsilon x-1+(\epsilon x+1) \cos (2 \theta)-2 \mathbf{E}\left(x_{\sigma}^{2}\right) \cos ^{3}(\theta) \sin (\theta) .
$$

Because $q\left(\frac{\pi}{2}\right)=-2 \neq 0$, Theorem 2 guarantees the existence of a unique normalized groundstate $\rho \in C^{\infty}\left(S_{\pi}^{1}\right)$. As $\alpha_{\frac{1}{3}, \sigma}=\imath x_{\sigma} / 2, \beta_{\frac{1}{3}, \sigma}=-\imath x_{\sigma} / 2$ and $\gamma_{\frac{1}{3}, \sigma}=x_{\sigma}^{2} / 2$, one deduces from Lemma 1 and Theorem 3 that up to errors of order $\mathcal{O}(\lambda)$

$$
\gamma(\lambda)=\lambda^{\frac{2}{3}}\left(\frac{1}{2}(\epsilon x+1) \int \mathrm{d} \theta \rho(\theta) \sin (2 \theta)+\frac{\mathbf{E}\left(x_{\sigma}^{2}\right)}{8} \int \mathrm{d} \theta \rho(\theta)(1+2 \cos (2 \theta)+\cos (4 \theta))\right),
$$


and, furthermore that $\mathcal{R}(\lambda)$ is, also up to errors of order $\mathcal{O}(\lambda)$, equal to

$$
\frac{\lambda^{\frac{2}{3}}}{2 \pi}\left(\epsilon x-1+(\epsilon x+1) \int \mathrm{d} \theta \rho(\theta) \cos (2 \theta)-\frac{\mathbf{E}\left(x_{\sigma}^{2}\right)}{4} \int \mathrm{d} \theta \rho(\theta)(2 \sin (2 \theta)+\sin (4 \theta))\right) .
$$

Let us remark that this formula is not the same as equation (35) of [DG], even though the invariant measure of $[\mathrm{DG}$ ] coincides with the above. In fact, their expansion of the expression $\log \left(1+\lambda^{2 / 3} t\right) \approx \lambda^{2 / 3} t$ is erroneous for large $t$.

\section{Appendix A: Transfer matrix at a band edge}

Let us consider a real analytic family $E \in \mathbb{R} \mapsto \mathcal{T}^{E} \in \mathrm{SL}(2, \mathbb{R})$ with

$$
\operatorname{Tr}\left(\mathcal{T}^{0}\right)=2,\left.\quad \partial_{E} \operatorname{Tr}\left(\mathcal{T}^{E}\right)\right|_{E=0} \neq 0 .
$$

If $\mathcal{T}^{E}$ is the transfer matrix of a periodic Jacobi matrix, this corresponds to a band edge at $E=0$. We show that necessarily the eigenvalue 1 of $\mathcal{T}^{0}$ has geometric multiplicity 1 , so that the Jordan form of $\mathcal{T}^{0}$ is non-trivial. A similar statement holds if $\operatorname{Tr}\left(\mathcal{T}^{0}\right)=-2$. For this purpose, let us choose the notation

$$
\mathcal{T}^{E}=\left(\begin{array}{ll}
a+A E & b+B E \\
c+C E & d+D E
\end{array}\right)+\mathcal{O}\left(E^{2}\right)
$$

Then one deduces $a d-b c=1, a+d=2$ and $A+D \neq 0$. Furthermore the order $E$ of $\operatorname{det}\left(\mathcal{T}^{E}\right)=1$ implies that $a D+d A-c B-b C=0$. We need to show that $\mathcal{T}^{0} \neq \mathbf{1}$. If $a \neq 1$, this is true. Hence suppose $a=1$ so that also $d=1$. Then $a d-b c=1$ implies that either $b=0$ or $c=0$. Hence $a D+d A-c B-b C=0$ implies that either $A+D-c B=0$ or $A+D-b C=0$. Because $A+D \neq 0$ it follows that either $c \neq 0$ or $b \neq 0$.

\section{Appendix B: Inhomogeneous singular first order ODE}

This appendix recollects some results about the solutions of the inhomogeneous first order ordinary differential equation (ODE) for a function $y=y(x)$ on the interval $U=(a, b)$

$$
p y^{\prime}+q y=r,
$$

where $p, q, r \in C^{m}(U)$ and $m \in \mathbb{N} \cup\{\infty\}$. In particular, we are interested in smooth solutions of the singular case where $p, q$ and $r$ have zeros at $\hat{x} \in U$ of order $0 \leq l_{p}, l_{q}<m$ and $l_{r}$ respectively. We suppose that $p$ and $q$ have no further zeros in $U$. If $l_{r}<\min \left\{l_{p}, l_{q}\right\}$, then there cannot be any solution $y \in C^{1}(U)$ because then

$$
\frac{p}{r} y^{\prime}+\frac{q}{r} y=1
$$

leads to the contradiction $0=1$ in the limit $x \rightarrow \hat{x}$. Hence let us suppose that $l_{r} \geq \min \left\{l_{p}, l_{q}\right\}$. In the regular case $l_{p} \leq l_{q}$, the homogeneous equation $p y^{\prime}+q y=0$ has a one parameter family 
of solutions $e^{-w}$ where $w(x)=\int^{x} \frac{q}{p} \in C^{m-l_{p}+1}(U)$ is any antiderivative. Then a one-parameter family of solutions of (43) is obtained by the method of variation of constants:

$$
y(x)=e^{-w(x)} \int^{x} \mathrm{~d} s e^{w(s)} \frac{r(s)}{p(s)},
$$

where the integral is well-defined because $l_{r} \geq l_{p}$. In the singular case, one has $l_{q}<l_{p}$ and $l_{q} \leq l_{r}$ and the zero of $p$ effectively decouples the intervals $(a, \hat{x})$ and $(\hat{x}, b)$. Any antiderivative $w(x)=\int^{x} \frac{q}{p}$ diverges as $x \rightarrow \hat{x}$. Nevertheless, we shall show below that these divergences may cancel out in (44). Let us note right away though that any solution $y \in C^{1}(U)$ has to satisfy

$$
y(\hat{x})=\lim _{x \rightarrow \hat{x}} \frac{r(x)}{q(x)} .
$$

This is a boundary condition for both intervals $(a, \hat{x})$ and $(\hat{x}, b)$. The question is whether there are differentiable solutions (at the point $\hat{x}$, in particular) and whether such a solution is unique, or there are one-parameter or two-parameter families of such solutions.

Proposition 3 Let $l_{r} \geq \min \left\{l_{q}, l_{p}\right\}$ and set $l=l_{p}-l_{q}$. Then the ODE (43) has continuous solutions $y$ with a number of free parameters as described in the following:

(i) If $l \leq 0$, there is a one-parameter family of solutions $y \in C^{m-l_{p}+1}(U)$.

(ii) If $l=1$ and $\partial \frac{p}{q}(\hat{x})>0$, the solution $y \in C^{m-l_{q}}(U)$ is unique.

(iii) If $l=1$ and $\partial \frac{p}{q}(\hat{x})<0$, then there is a two-parameter family of solutions $y \in C^{n}(U)$ as

long as $n \leq m-l_{q}$ and $n<\left|\partial \frac{p}{q}(\hat{x})\right|^{-1}$.

(iv) If $l \geq 2$ is even and $\partial^{l} \frac{p}{q}(\hat{x})>0$, the solution $y \in C^{m-l_{q}}(U)$ is unique for $x>\hat{x}$, but there is one free parameter for $x<\hat{x}$.

(v) If $l \geq 2$ is even and $\partial^{l} \frac{p}{q}(\hat{x})<0$, the solution $y \in C^{m-l_{q}}(U)$ is unique for $x<\hat{x}$, but there is one free parameter for $x>\hat{x}$.

(vi) If $l \geq 3$ is odd and $\partial^{l} \frac{p}{q}(\hat{x})>0$, the solution $y \in C^{m-l_{q}}(U)$ is unique.

(vii) If $l \geq 3$ is odd and $\partial^{l} \frac{p}{q}(\hat{x})<0$, there is a two-parameter family of solutions $y \in C^{m-l_{q}}(U)$.

Any other solution $y$ has a non-integrable singularity at $\hat{x}$.

Proof. In case (i) the solutions are given in (44). Next let us consider the case (iv). Dividing (43) by $q$ gives $\frac{p}{q} y^{\prime}+y=\frac{r}{q}$. Hence it is sufficient to consider the case $p y^{\prime}+y=r$ with $p, r \in C^{m-l_{q}}(U)$ and $l_{p}=l$. Let $w$ be any antiderivative of $\frac{1}{p}$ on $U \backslash\{\hat{x}\}$. Because of $\partial^{l} p(\hat{x})>0$ and $l$ is even, one has $\lim _{x \uparrow \hat{x}} w(x)=+\infty$ and $\lim _{x \downarrow \hat{x}} w(x)=-\infty$. Moreover, the growth of $|w(x)|$ is at least as $|x-\hat{x}|^{-1}$ because $l \geq 2$. Thus for all $n \in \mathbb{N}$

$$
\lim _{x \uparrow \hat{x}} p^{n}(x) e^{w(x)}=+\infty, \quad \lim _{x \downarrow \hat{x}} \frac{e^{w(x)}}{p(x)}=0 .
$$

Now $e^{-w}$ is a solution of the homogeneous equation $p y^{\prime}+y=0$. Because $\lim _{x \downarrow \hat{x}} w(x)=-\infty$, the only possible continuous solution of $p y^{\prime}+y=r$ for $x>\hat{x}$ is

$$
y(x)=e^{-w(x)} W(x), \quad W(x)=\int_{\hat{x}}^{x} \mathrm{~d} s e^{w(s)} \frac{r(s)}{p(s)},
$$


where the last integral exists due to (46). For $x<\hat{x}$, let $W$ be any antiderivative of $e^{w} \frac{r}{p}$ and also set $y=e^{-w} W$. Hence $y$ is now a solution of $p y^{\prime}+y=r$ for $x \neq \hat{x}$. It remains to be shown that $y \in C^{m-l_{q}}(U)$. First of all, $y$ is continuous because

$$
\lim _{x \rightarrow \hat{x}} y(x)=\lim _{x \rightarrow \hat{x}} \frac{W(x)}{e^{w(x)}}=\lim _{x \rightarrow \hat{x}} \frac{e^{w(x)} \frac{r(x)}{p(x)}}{e^{w(x)} \frac{1}{p(x)}}=r(\hat{x})=y(\hat{x}),
$$

the latter by (45). In the second equality, de l'Hospital's rule could be applied because for $x \downarrow \hat{x}$ the numerator and denominator both converge to 0 by (46) and (47), while for $x \uparrow \hat{x}$ as the denominator converges to $\infty$ by (46).

Next follows an inductive argument in order to check the continuity of the higher derivatives of $y$ at $\hat{x}$. Let us set:

$$
q_{n}=n p^{\prime}+1, \quad r_{n}=r_{n-1}^{\prime}-q_{n-1}^{\prime} y^{(n-1)}, \quad r_{0}=r
$$

Note that $q_{n}$ has no zero at $\hat{x}$. One can check by induction

$$
p y^{(n+1)}+q_{n} y^{(n)}=r_{n} .
$$

Let $w_{n}=w+n \log (|p|)$. Now $e^{-w_{n}}$ is a solution of the homogeneous equation $p y^{(n+1)}+q_{n} y^{(n)}=$ 0 and satisfies due to (46)

$$
\lim _{x \uparrow \hat{x}} p(x) e^{w_{n}(x)}=+\infty, \quad \lim _{x \downarrow \hat{x}} e^{w_{n}(x)}=0
$$

Now let $y^{(n)}, n<m-l_{g}$, be continuous by induction hypothesis. Then $r_{n} \in C^{1}(U)$. Set $W_{n}=y^{(n)} e^{w_{n}}$. Due to (49), one has $\lim _{x \downarrow \hat{x}} W_{n}(x)=0$. Using the identities

$$
y^{(n+1)}=\frac{r_{n} e^{w_{n}}-q_{n} W_{n}}{e^{w_{n}} p}, \quad q_{n} W_{n}^{\prime}=r_{n} w_{n}^{\prime} e^{w_{n}}
$$

which follow from (48) and $w_{n}^{\prime}=\frac{q_{n}}{p}$, one obtains

$$
\begin{aligned}
\lim _{x \rightarrow \hat{x}} y^{(n+1)}(x) & =\lim _{x \rightarrow \hat{x}} \frac{-q_{n}(x) W_{n}(x)+r_{n}(x) e^{w_{n}(x)}}{e^{w_{n}(x)} p(x)} \\
& =\lim _{x \rightarrow \hat{x}} \frac{-q_{n}^{\prime}(x) W_{n}(x)+r_{n}^{\prime}(x) e^{w_{n}(x)}}{e^{w_{n}(x)}\left(p^{\prime}(x)+q_{n}(x)\right)}=\frac{-q_{n}^{\prime}(\hat{x}) y^{(n)}(\hat{x})+r_{n}^{\prime}(\hat{x})}{q_{n+1}(\hat{x})}
\end{aligned}
$$

where de l'Hospital's rule can be used for $x \downarrow \hat{x}$, because the numerator and denominator converge both to 0 according to (49) and for $x \uparrow \hat{x}$ as the denominator converges to $\pm \infty$. Therefore $y^{(n)}$ is continuously differentiable in $\hat{x}$.

The case $(\mathrm{v})$ is identical, once the sides $x>\hat{x}$ and $x<\hat{x}$ are exchanged in the above argument. In case (vi), one has $\lim _{x \rightarrow \hat{x}} w(x)=-\infty$ (namely, for both $x \downarrow \hat{x}$ and $x \uparrow \hat{x}$ ), so that one has to construct the solution as in (47) both for $x>\hat{x}$ and $x<\hat{x}$; hence the solution 
is unique. In case (vii), one has $\lim _{x \rightarrow \hat{x}} w(x)=+\infty$ and the homogeneous solutions on both sides vanish at $\hat{x}$ faster than any power, so that one has a two-parameter family of solutions.

Finally let us consider $l=1$ and first the case (ii). Hence $\lim _{x \rightarrow \hat{x}} w(x)=-\infty$ so that $\lim _{x \rightarrow \hat{x}} e^{w(x)}=0$. Now the second limit in (46) may diverge to $+\infty$, but one readily checks that $\frac{e^{w}}{p}$ is integrable so that $W$ can be defined as in (47) for $x>\hat{x}$ and $x<\hat{x}$. Now the continuity is proven as above, and higher derivatives can also be treated as above because

$$
\lim _{x \rightarrow \hat{x}} e^{w_{n}(x)}=0
$$

so that both nominator and numerator of $y^{(n+1)}$ converge to 0 and de l'Hopital's rule may be applied as before.

In case (iii), $\lim _{x \rightarrow \hat{x}} w(x)=\infty$ so that $\lim _{x \rightarrow \hat{x}} e^{-w(x)}=0$. Furthermore $\frac{e^{w}}{p}$ is never integrable and the two-parameter family of solutions $y$ is defined as in (44). The main point to note is that $w_{n}=\left(p^{\prime}(\hat{x})^{-1}+n\right) \log (|x-\hat{x}|)+\mathcal{O}(1)$, hence

$$
\lim _{x \uparrow \hat{x}} p(x) e^{w_{n-1}(x)}=\infty, \quad \lim _{x \downarrow \hat{x}} p(x) e^{w_{n-1}(x)}=-\infty
$$

under the hypothesis stated in (iii). Thus de l'Hopital's rule can be invoked to calculate $\lim _{x \rightarrow \hat{x}} y^{(n)}$ as above (with the index $n$ shifted by 1 ). Let us remark that in case $\left|p^{\prime}(\hat{x})\right|^{-1} \in \mathbb{N}$, one can prove better differentiability, but only for a one-parameter family of solutions (just as for the ODE $\left.x y^{\prime}=N y, N \in \mathbb{N}\right)$.

The singularity of all other solutions is at least of the type $|x-\hat{x}|^{-1}$ in cases (ii) to (vii).

\section{References}

[BL] P. Bougerol, J. Lacroix, Products of Random Matrices with Applications to Schrödinger Operators, (Birkhäuser, Boston, 1985).

$[\mathrm{BK}]$ A. Bovier, A. Klein, Weak disorder expansion of the invariant measure for the onedimensional Anderson model, J. Stat. Phys. 51, 501-517 (1988).

[CK] M. Campanino, A. Klein, Anomalies in the one-dimensional Anderson model at weak disorder, Commun. Math. Phys. 130, 441-456 (1990).

[DG] B. Derrida, E. J. Gardner, Lyapunov exponent of the one dimensional Anderson model: weak disorder expansion, J. Physique 45, 1283-1295 (1984).

[JSS] S. Jitomirskaya, H. Schulz-Baldes, G. Stolz, Delocalization in random polymer chains, Commun. Math. Phys. 233, 27-48 (2003).

[KW] M. Kappus, F. Wegner, Anomaly in the band centre of the one-dimensional Anderson model, Z. Phys. B 45, 15-21 (1981). 
[PF] L. Pastur, A. Figotin, Spectra of Random and Almost-Periodic Operators, (Springer, Berlin, 1992).

[Ris] H. Risken, The Fokker-Planck equation, Second Edition, (Springer, Berlin, 1988).

[S1] H. Schulz-Baldes, Lyapunov exponents at anomalies of $\mathrm{SL}(2, \mathbb{R})$ actions, to be publ. in Operator Theory, Advances and Applications

[S2] H. Schulz-Baldes, Lifshitz tails for the 1D Bernoulli-Anderson model, Markow Processes and Related Fields 9, 795-802 (2004).

[SSS] R. Schrader, H. Schulz-Baldes, A. Sedrakyan, Perturbative test of single parameter scaling for $1 D$ random media, Ann. H. Poincare 5, 1159-1180 (2004).

[SVW] C. Shubin, R. Vakilian, T. Wolff, Some harmonic analysis questions suggested by Anderson-Bernoulli models, Geom. Funct. Anal. 8 932-964 (1998).

[Tho] D. J. Thouless, in Ill-Condensed Matter, Les Houches Summer School, 1978, edited by R. Balian, R. Maynard, G. Toulouse (North-Holland, New York, 1979). 